\title{
Cinder cone morphometry in relation to gravity anomaly zones in the Harrat al Birk and Asir foreland, SW Saudi Arabia
}

\author{
Basab Mukhopadhyay ${ }^{1, *}$, Manoj Mukhopadhyay ${ }^{2}$, Eslam Elawadi ${ }^{3}$, \\ Uday Kumar Ghosh ${ }^{4}$ and KaUshik PramaniK ${ }^{4}$ \\ ${ }^{1}$ Map and Cartography Division, Geological Survey of India, 29 J.L. Nehru Road, Kolkata 700 016, India. \\ ${ }^{2}$ Department of Applied Physics, University of Technology, Lae, Morobe Province, Papua New Guinea. \\ ${ }^{3}$ Deanship of Scientific Research, King Saud University, P.O. Box 2455, Riyadh 11451, Kingdom of Saudi Arabia. \\ ${ }^{4}$ PGRS Division, Geological Survey of India, 29 J.L. Nehru Road, Kolkata 700 016, India. \\ *Corresponding author.e-mail: basabmukhopadhyay@gmail.com
}

MS received 6 July 2018; revised 19 February 2019; accepted 26 February 2019; published online 4 June 2019

The Harrat al Birk (HAB) of the alkali basalts of the post-Miocene age erupted through a thick Precambrian crust along the Red Sea coast. The 200 cinder cones scatter within HAB and three distinct Bouguer anomaly zones are identified as the coastal gravity high (CGH), axial gravity low (AGL) and a gravity high further east Jabal As-Sawda gravity high (JSGH). Interpreted satellite imagery for 150 sizeable cinder cones in three gravity anomaly zones (134 (90\%) in CGH, 10 (6\%) in AGL and 6 (4\%) in JSGH) are morphologically analysed. The juvenile cinder cones (age $<1 \mathrm{Ma}$ ) are characterised by subvertical slope angles $\left(<40^{\circ}\right)$ with a variable average geomorphic slope, $27^{\circ}-40^{\circ}$ in $\mathrm{JSGH}, 25^{\circ}$ in $\mathrm{AGL}$ and $2^{\circ}-15^{\circ}$ in $\mathrm{CGH}$. The oldest cones of JSGH display a high $H_{\mathrm{co}} / W_{\text {co }}$ ratio with angular asymmetry at their base angles. The major feeder lineaments/faults are oriented NNW-SSE to NW-SE, and they supply volcanic material to cinder cones within a distance of $2 \mathrm{~km}$. The results, when integrated with geological maps and gravity profiles, provide a probable root-plumbing system of the volcano edifice. $\mathrm{HAB}$ is the produce of the volcanic process from the source area in JSGH that hosts the major cinders, where the tertiary gabbroic dyke zone acts as a vertical magma sheet for the cinders.

Keywords. Cinder cone morphometry; Harrat al Birk; Asir foreland; DEM; TGDZ; root-plumbing system.

\section{Introduction}

The Harrat al Birk (HAB) (harrat means volcanic fields and flows) presents an excellent opportunity to examine the Red Sea continental margin volcanism in SW Saudi Arabia. The unique feature of the HAB is that it represents volcanism from Tertiary through the Cenozoic concomitant to rifting (Bosworth et al. 2005) where volcanism clearly continued uninhibited through historical times (figure 1). The Ad-Darb transform (ADT) defines the contact zone between the Miocene crust to the south of HAB against the Precambrian crust to the north where the basalts of HAB erupted in the Quaternary period. Many of the volcanic fields in HAB were initially mapped by Ghent et al. (1980), Hadley (1982) and Coleman et al. (1983). The HAB and other smaller harrats along the southern Red Sea coast are amongst the Cenozoic volcanic fields belonging to the large 


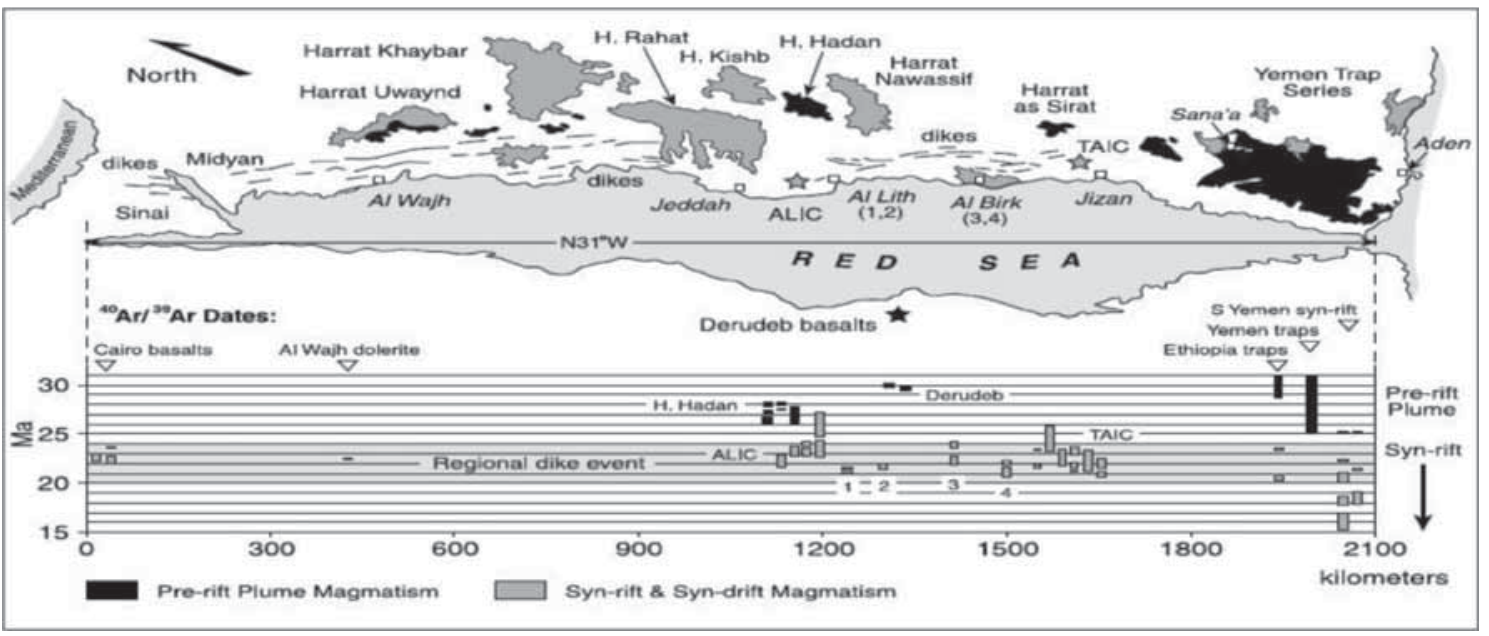

Figure 1. Compiled ${ }^{40} \mathrm{Ar} /{ }^{39} \mathrm{Ar}$ dates for the Red Sea basin, and location of major tertiary magmatism in Saudi Arabia, including the HAB at the Red Sea Coast, their eruption ages (compiled after: Bosworth et al. 2005; Németh et al. 2014). Numbers 1-4 refer to gabbroic dike localities at Al-lith and Al-Birk; ALIC, the Al-lith igneous complex and the TAIC, Tihama Asir igneous complex in Saudi Arabia. For other notations, refer Bosworth et al. (2005).

igneous province in Saudi Arabia that are being investigated with renewed interest in recent years (Inglis et al. 2013, 2014a, b; Németh et al. 2014). We have presented morphometric analysis on the cinder cones (by definition the cinder cones are steep conical hill formed by the accumulation of glassy volcanic fragments around a volcanic vent) based on satellite imagery to study their eruptive character in the large igneous province in Saudi Arabia (Zaidi and Mukhopadhyay 2015). Results on crustal investigation using the deep seismic refraction method by the United States Geological Survey (USGS) (Healy et al. 1982; Mooney et al. 1985) and their combined interpretation using geologic and gravity data (Schimdt et al. 1983; Gettings et al. 1986) led to an overall understanding on the crustal configuration under the Arabian shield and continent to ocean crustal transition under the southern Red Sea continental margin in SW Saudi Arabia. The present study focuses on the area further north of it where the HAB Quaternary basalts erupted encompassing an area of $1800 \mathrm{~km}^{2}$; it is typically $30 \mathrm{~km}$ wide in the east-west direction. Bohannon et al. (1989) have given an account of the timing of uplift and volcanism relating to Red Sea rifting. The area is tectonically active as evidenced by its present seismicity (Merghelani and Gallanthine 1980; Abdelfattah et al. 2017).

Our contention here is to estimate the morphometric parameters, which in turn provide the quantitative description of the cinder cones in HAB and adjoining areas, and to study their variation in relation to the three contrasting gravity anomaly zones which occupy the area. We interpret the gravity field further by a long gravity profile across the Harrat. Here, we shall rather emphasise how the tertiary gabbroic dyke zone (TGDZ) situated between the two conspicuous gravity anomaly zones influence the magmatic source and the eruptive process through our proposed volcanostructural and root-plumbing process analysis.

\section{Volcano-structural analysis for the HAB and Asir foreland}

Two prominent wadis (stream), Wadi Hali and Wadi Nahb, mostly outline the extent of the elongated harrat from the pediment of the Asir mountains (figure 2). HAB basalts erupted over an area of $1800 \mathrm{~km}^{2}$; having a width of $30 \mathrm{~km}$ in the east-west direction (Coleman et al. 1983). Past the TGDZ, two juvenile craters at a gap of $58 \mathrm{~km}$ at Jabal Al-Haylah and Jabal Bagarah along the east margin of the HAB exist; but their relationship to the main harrat remains uninvestigated. In the following sections, we shall present evidences based on gravity anomalies how they correlate to the main part of HAB. Several geological features make this region distinct: (a) the juvenile age of volcanism continuing into the present (ages <2Ma), (b) volcanism along the Red Sea coast producing an elliptical-shaped harrat whose east margin is dissected by TGDZ of continental affinity, (c) a regionally extensive tectonic lineament, called the Red Sea Escarpment lies further 


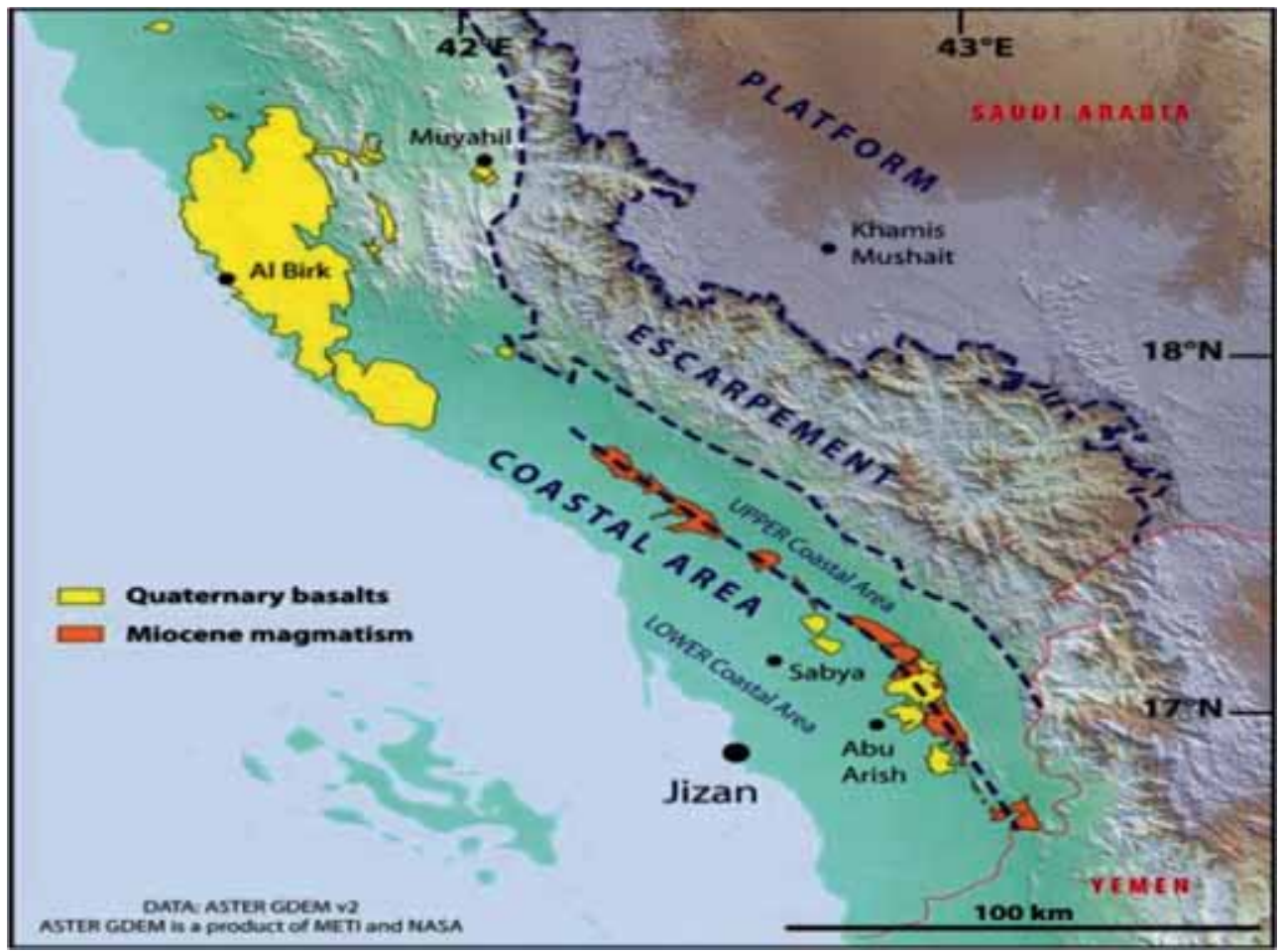

Figure 2. Geologic outline for the HAB and As-Sarawat mountains in SW Saudi Arabia (redrawn after Devès et al. 2013); the harrat forms a part of the large igneous province in Saudi Arabia (Németh et al. 2014; Zaidi and Mukhopadhyay 2015).

to the east bordering the Foothills fault system; the latter defines the contact between the HAB volcanic fields against the foothills of the Asir mountains bordering the Arabian shield. This physiographic escarpment has been interpreted in different ways: (i) based on the USGS seismic experiment, Mooney et al. (1985) interpreted it as the surface trace of a dipping seismic layer between the transitional crust south of it against the Arabian shield crust, (ii) field geologic mapping by USGS (Bohannon et al. 1989) postulated it as the zone of the tertiary mafic crust and (iii) based on gravity modelling results, we have argued elsewhere for a rather wide transitional crust of greater density below the escarpment (Mogren and Mukhopadhyay 2013). The unique feature of the ADT is that it defines the contact zone between the Miocene crust to its south against the Precambrian crust to the north where the HAB basalts erupted in the Quaternary.

As many as 200 cinder cones are mapped within $\mathrm{HAB}$, of which 150 cinders are better manifested. They follow a northwesterly trend but are now deeply dissected in central lava vents (figure 3). Their original appearance might have been $0.8^{-}$ 1.5 inch diameter, of height less than $0.15 \mathrm{~km}$ (Coleman et al. 1983). Most of the cinder cones are rather small-size craters which were breached by central-vent lava flows. Xenoliths are commonly found in the cinder cones and also within the solidified flows; they are inferred to originate from the lower crust. TGDZ separates the elliptical HAB from the juvenile cinder cones at Jabal Al-Haylah and Jabal Baqarah, respectively (Jabal indicates mountain). These two cinder cones are $\sim 57 \mathrm{~km}$ apart striking north-south; their respective locations are: Jabal Al-Haylah (N18 $31^{\prime} 2.42^{\prime \prime}$; E $42^{\circ} 2^{\prime} 56.15^{\prime \prime}$; elevation $710 \mathrm{~m}$ ) and Jabal Baqarah (N22 $56^{\prime} 48^{\prime \prime}$; E42 ${ }^{\circ} 46^{\prime} 11^{\prime \prime}$; average elevation $362 \mathrm{~m}$ ) (figure 4). The surface width of the TGDZ varies from $14 \mathrm{~km}$ to the west of the Jabal Baqarah but widening to almost $25 \mathrm{~km}$ in the direction of the Jabal Al-Haylah.

The gravity data on a $10-\mathrm{km}$ square grid involving 2196 stations covering $202,100 \mathrm{~km}^{2}$ in SW Saudi Arabia from USGS (Gettings et al. 1986) have been used. Figure 5 shows the gravity station coverage and the contoured Bouguer anomaly (BA) map as an overlay on surface geology. Notice that the gravity survey lines within the study area are mostly oriented NE-SW, traversing orthogonal to the structural trend of the HAB. But some gravity survey lines are NW-SE to N-S directed and run locally subparallel to the Red Sea coast. The BA 


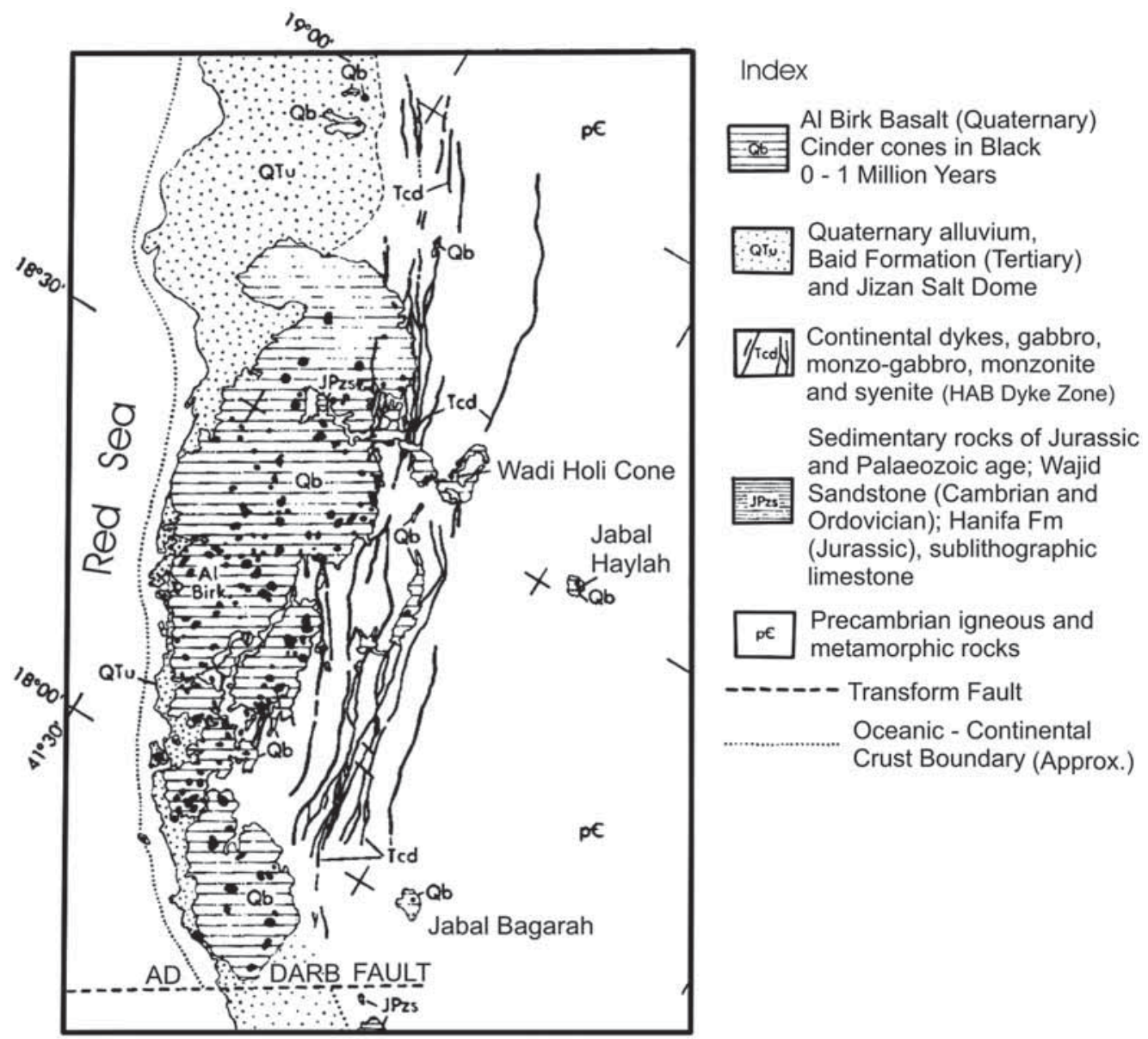

Figure 3. Quaternary basalts forming the HAB and the two most conspicuous cinder cones at the Jabal Al-Haylah and the Jabal Bagarah along the north margin of the harrat; redrawn after Ghent et al. (1980). The ADT fault dissects the southern margin of the harrat at about $17.5^{\circ} \mathrm{N}$ latitude.

map reveals three principal anomaly trends in the area:

(a) Coastal gravity high (CGH) running all along the Red Sea coast and extending some $15-\mathrm{km}$ distance inland. Anomaly amplitude in CGH ranges from -15 to +27 mgal, exhibiting a steep gravity gradient towards the shoreline.

(b) Elongated zone of low gravity, trending NNW, further inland labelled as axial gravity low (AGL) developed between CGH and the AsSarawat mountains west of Abha (a renowned tourist spot in SW Saudi Arabia) with a peak gravity low reaches a value of -93 mgal. It is a wider expanse of low gravity, interrupted by an east-west trending strip of narrow gravity high passing south of the Jabal Hayilah.

(c) Zone of relative gravity high, here referred to as the Jabal As-Sawda gravity high (JSGH), striking NW, that passes between the AGL and the Asir mountains to the east of it. JSGH not only delimits the AGL but also transgresses it as the tapered JSGH is developed coastward across the Jabals at Tusi Al-Yamani and at Tusi Ash Shami.

These three gravity anomaly zones indicate crustal heterogeneity within the continent to the ocean transitional crust of higher density under the southern Red Sea continental margin. The correlation of these gravity zones with a prominent geological structure is yet to be established.

TGDZ of figure 5 (also referred to as the HAB dyke zone or tcd in figures 3 and 4) is represented as a dyke swarm system of average width of $\sim 20 \mathrm{~km}$. These sheeted dykes are composed of primarily gabbro (with clinopyroxene, plagioclase with accessory magnetite, chlorite and apatite with \pm hornblende) yielded an age 19-27 Ma (K/Ar whole rock age), and are intruded close to the initial opening of the Red Sea rift (Hadley 1982). These parallel normal faults along the sheeted dyke zones generally follow the north to the north-west trend, and in our analysis, these normal 


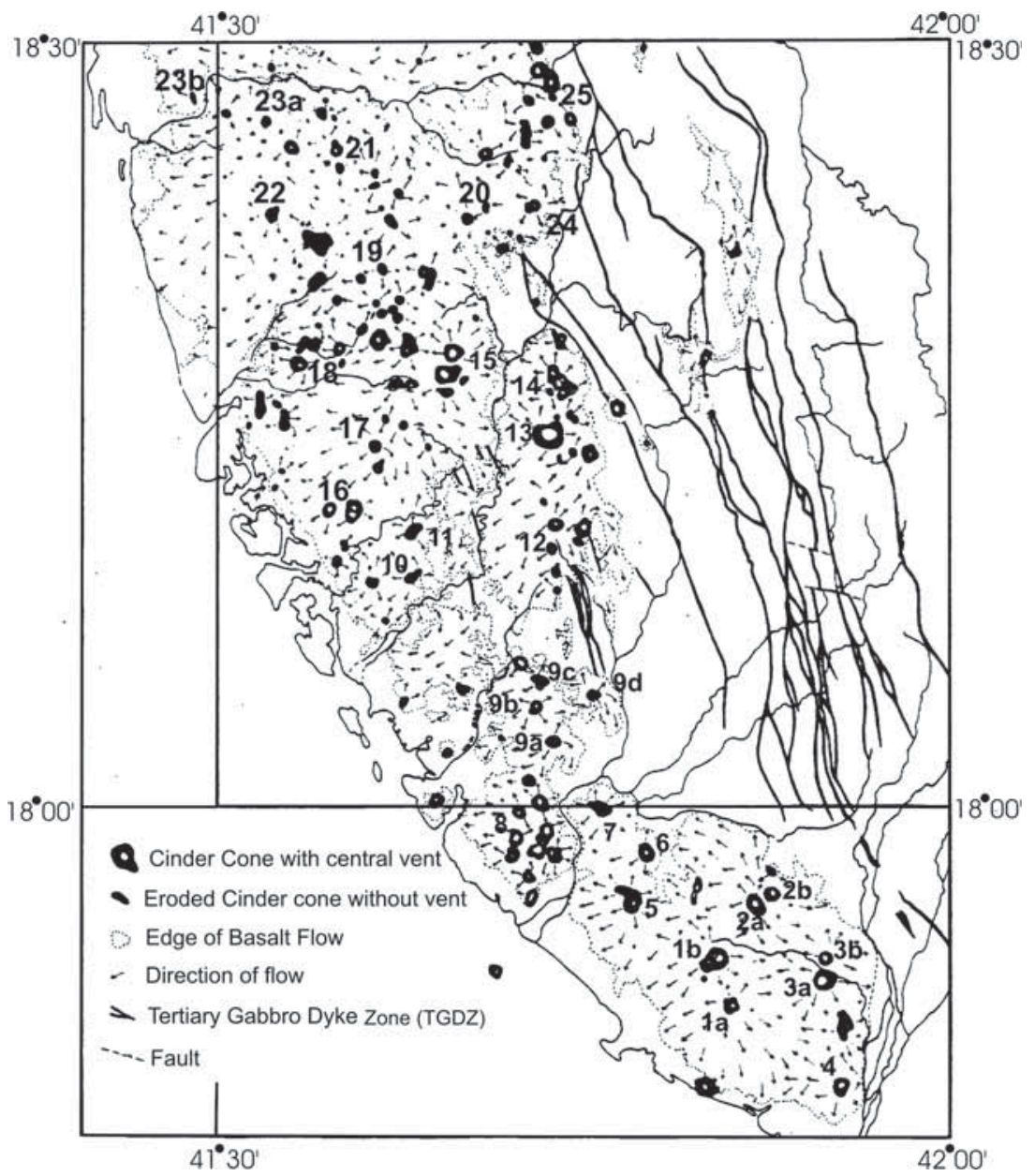

Figure 4. Distribution of as many as 30 sizeable cinders in the Wadi Dhahaban Quadrangle and adjoining areas in the western parts of the HAB at the Red Sea margin, the TGDZ defines their eastern limit. The base map is after Hadley (1982).

faults and subsidiary fault zones within the crustal level act as major feeder channels for the volcanoes in the respective gravity anomaly zones of CGH, AGL and JSGH.

\section{Morphometric analysis of cinder cones based on satellite data}

Morphometric analysis for the cinders considered here is grouped below in three categories as per their geographic locations, geologic affinity and associated gravity anomaly zones: CGH, AGL and JSGH. The analysis is carried out using the freely available 30-m ASTER digital elevation model (DEM) and an enhanced thematic mapper $(\mathrm{ETM})+$ image. Based on the multispectral Landsat ETM+, the scorias are demarcated and fused with a shaded relief map. A slope map is generated using the ENVI 4.8 software, while the morphometric investigations are executed in the ArcGIS platform. A total of 150 cinder cones have been identified based on the image and DEM analysis which are of different dimensions. Certain constraints are experienced during morphometric analysis due to the resolution issue associated with the ASTER GDEM - version 2 data having a maximum resolution of $30 \mathrm{~m}$ with a vertical accuracy of $8.68 \mathrm{~m}$ (Taachikawa et al. 2011). Despite such limitations, it is possible to define the basic relationship between the morphometric parameters for the 134 cinder cones for CGH, 10 cinder cones pertaining to AGL and 6 cinder cones belonging to JSGH (table 1).

The cinder cones are initially identified from the satellite images (ETM+) taking the help of the Google Earth application. This selection is based on two criteria: (i) regular symmetrical shape and (ii) their easy distinction from the surroundings based on an abrupt change in slope. The ASTER 


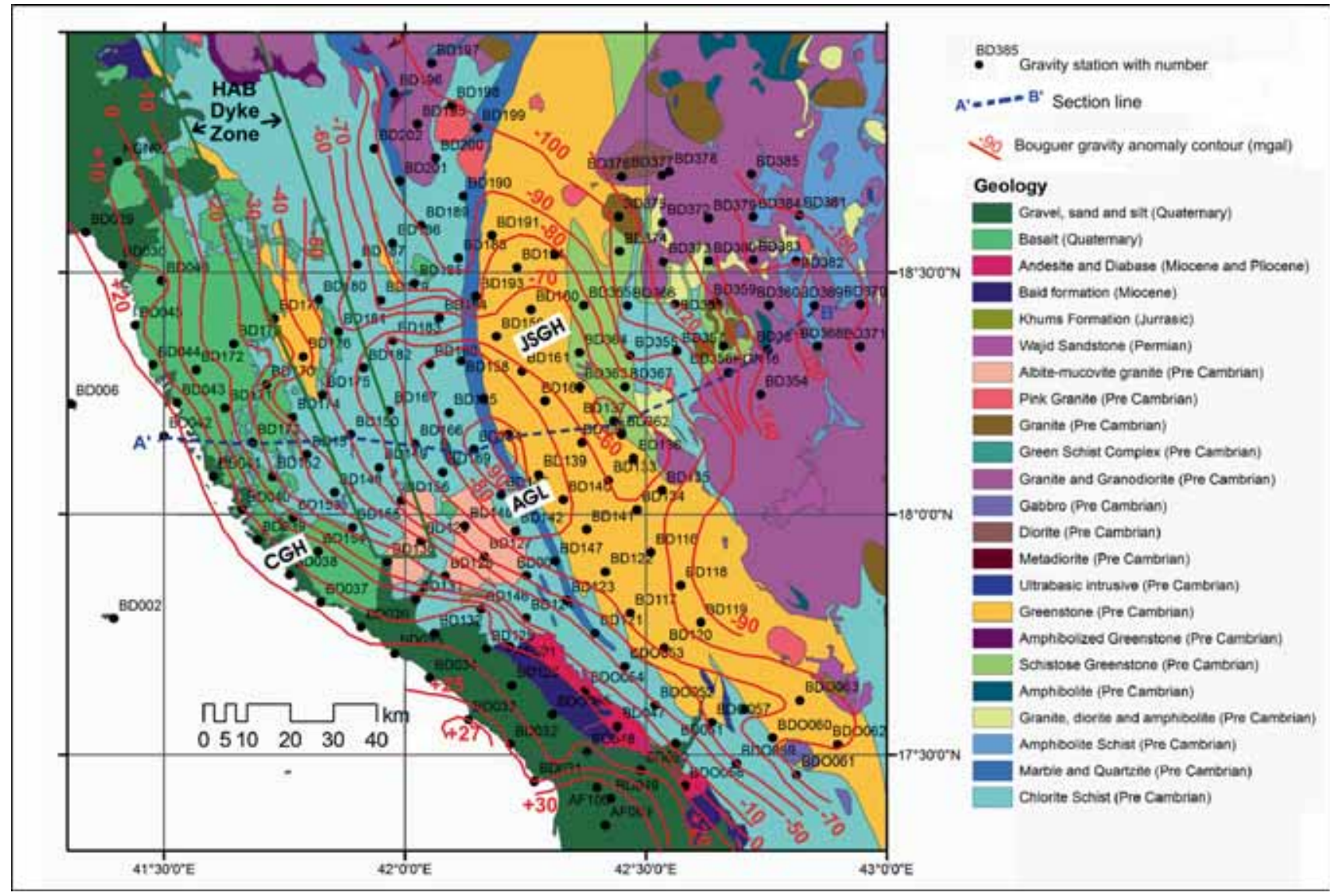

Figure 5. Bouguer gravity anomaly map for the HAB and the Asir foreland in areas of the As-Sarawat mountains, with gravity station locations and surface geology as overlay. 2D-gravity interpretation suggestive of the source model (along $\mathrm{A}^{\prime}-\mathrm{B}^{\prime}$ ) below the harrat is discussed in the following section. CGH: coastal gravity high, AGL: axial gravity low, JSGH: Jabal As-Sawda gravity high.

DEM which provides 1 arcs (30 $\mathrm{m}$ resolution) data in a geographic latitude-longitude coordinate system was downloaded for the present study and the subsequent measurements are made, such as the cone basal diameter $\left(W_{\mathrm{co}}\right)$, cone height $\left(H_{\mathrm{co}}\right)$, crater diameter $\left(W_{\text {cr }}\right)$, cone slope and cone volume and their aspect ratios, $H_{\mathrm{co}} / W_{\mathrm{co}}$ and $W_{\mathrm{cr}} / W_{\mathrm{co}}$. The measurements are carried out in ArcGIS 10.3 and the ENVI 4.8 software and tabulated zonewise (see table 1).

The 3D topographic model (figure 6a) and corresponding slope map (figure $6 \mathrm{~b}$ ) generated in the ENVI environment from the available DEM of the volcanic fields helps in delineating the cone diameter. The cone base (diameter) is then calculated manually with the help of image interpretation, DEM and contour. In the case of the elongated cones, the long and short axes of the delineated cone diameter were considered and the average value was taken as the representative cone diameter. For calculating the cone height, the contour value slope map (up to $2^{\circ}$ values) and
DEM data were taken into consideration which provide the difference between the maximum and the minimum values. For calculating the crater diameter contour, we consider the shaded relief map and Google image. It is intriguing to observe that their average slope in CGH is very low $\left(<8^{\circ}\right)$, but it increases to $8^{\circ}-16^{\circ}$ in AGL and still further increases to $16^{\circ}-36^{\circ}$ in JSGH (figure $6 \mathrm{~b}$ ).

\subsection{Cinder cone morphometry in correspondence to gravity anomaly zones of the $C G H$}

Prominent cinders located within CGH are the Jabal at Tusi Ash Shami (marked as No. 1), Jabal at Tusi Al-Yamani (No. 2), Jabal Qurayn (No. 3) and Al-Birk Cinder (No. 4); the last one is regarded as the main eruptive centre in the region (figure 7 ). Larger cinder cones in HAB are Jabal at Tusi Ash Shami, Jabal at Tusi Al-Yamani, Jabal Qurayn located near the coast; whereas the cinders at Jabal Al-Qishr, Jabal Ba'a, Jabal Al-Hayilah and Jabal Baqarah are located further inland. Most of the 
Table 1. Morphometric estimates for the cinder cones in the Harrat Al-Birk and the Asir foreland in the zones.

\begin{tabular}{|c|c|c|c|c|c|c|c|c|c|}
\hline Zone & $\begin{array}{c}\text { Cone } \\
\text { no. }\end{array}$ & Long. & Lat. & $\begin{array}{l}\text { Cone_basal } \\
\left(W_{\text {co }}\right)(\mathrm{m})\end{array}$ & $\begin{array}{c}\text { Cone_heigh } \\
\left(H_{\mathrm{co}}\right)(\mathrm{m})\end{array}$ & $\begin{array}{l}\text { Crater_dia } \\
\left(W_{\mathrm{cr}}\right)(\mathrm{m})\end{array}$ & $H_{\mathrm{co}} / W_{\mathrm{co}}$ & $W_{\mathrm{cr}} / W_{\mathrm{co}}$ & $\begin{array}{l}\text { Volume } \\
\left(10^{6} \mathrm{~m}^{3}\right)\end{array}$ \\
\hline CGH & $3 b$ & 41.9191 & 17.9043 & 871.00 & 40.00 & 284.00 & 0.05 & 0.33 & 7.94 \\
\hline $\mathrm{CGH}$ & 5 & 41.7854 & 17.9400 & 1825.00 & 140.00 & 568.00 & 0.08 & 0.31 & 122.01 \\
\hline $\mathrm{CGH}$ & $2 \mathrm{~b}$ & 41.8794 & 17.9459 & 1447.00 & 120.00 & 514.00 & 0.08 & 0.36 & 65.75 \\
\hline $\mathrm{CGH}$ & $2 \mathrm{a}$ & 41.8697 & 17.9357 & 1427.00 & 160.00 & 365.00 & 0.11 & 0.26 & 85.25 \\
\hline $\mathrm{CGH}$ & 6 & 41.7944 & 17.9715 & 1353.00 & 100.00 & 400.00 & 0.07 & 0.30 & 47.90 \\
\hline $\mathrm{CGH}$ & 8 & 41.7053 & 17.9836 & 1395.00 & 160.00 & 192.00 & 0.11 & 0.14 & 81.47 \\
\hline $\mathrm{CGH}$ & $9 \mathrm{a}$ & 41.7308 & 18.0428 & 999.00 & 100.00 & 173.00 & 0.10 & 0.17 & 26.11 \\
\hline $\mathrm{CGH}$ & $9 \mathrm{~b}$ & 41.7193 & 18.0650 & 1025.00 & 120.00 & 190.00 & 0.12 & 0.19 & 32.99 \\
\hline $\mathrm{CGH}$ & $9 \mathrm{c}$ & 41.7222 & 18.0812 & 1295.00 & 140.00 & 191.00 & 0.11 & 0.15 & 61.44 \\
\hline $\mathrm{CGH}$ & 10 & 41.6092 & 18.1470 & 926.00 & 80.00 & 194.00 & 0.09 & 0.21 & 17.95 \\
\hline $\mathrm{CGH}$ & 11 & 41.6348 & 18.1808 & 892.00 & 80.00 & 174.00 & 0.09 & 0.20 & 16.66 \\
\hline $\mathrm{CGH}$ & 16 & 41.6103 & 18.2261 & 827.00 & 120.00 & 208.00 & 0.15 & 0.25 & 21.48 \\
\hline $\mathrm{CGH}$ & 17 & 41.6456 & 18.2265 & 523.00 & 20.00 & 133.00 & 0.04 & 0.25 & 1.43 \\
\hline $\mathrm{CGH}$ & 19 & 41.5997 & 18.3422 & 721.00 & 40.00 & 204.00 & 0.06 & 0.28 & 5.44 \\
\hline $\mathrm{CGH}$ & $23 \mathrm{~b}$ & 41.4788 & 18.4768 & 492.00 & 20.00 & 126.00 & 0.04 & 0.26 & 1.27 \\
\hline $\mathrm{CGH}$ & 21 & 41.6084 & 18.4090 & 515.00 & 20.00 & 125.00 & 0.04 & 0.24 & 1.39 \\
\hline $\mathrm{CGH}$ & $23 \mathrm{a}$ & 41.5519 & 18.4410 & 1259.00 & 120.00 & 271.00 & 0.10 & 0.22 & 49.77 \\
\hline $\mathrm{CGH}$ & 26 & 41.6159 & 18.3049 & 1877.00 & 200.00 & 169.00 & 0.11 & 0.09 & 184.38 \\
\hline $\mathrm{CGH}$ & 20 & 41.6711 & 18.3804 & 1207.00 & 140.00 & 196.00 & 0.12 & 0.16 & 53.37 \\
\hline $\mathrm{CGH}$ & 24 & 41.7150 & 18.3882 & 1137.00 & 60.00 & 295.00 & 0.05 & 0.26 & 20.30 \\
\hline $\mathrm{CGH}$ & 25 & 41.7391 & 18.4454 & 1040.00 & 40.00 & 121.00 & 0.04 & 0.12 & 11.32 \\
\hline $\mathrm{CGH}$ & 15 & 41.6616 & 18.2929 & 845.00 & 80.00 & 203.00 & 0.09 & 0.24 & 14.95 \\
\hline $\mathrm{CGH}$ & 13 & 41.7259 & 18.2415 & 1909.00 & 120.00 & 270.00 & 0.06 & 0.14 & 114.43 \\
\hline $\mathrm{CGH}$ & 14 & 41.7290 & 18.2809 & 1680.00 & 120.00 & 419.00 & 0.07 & 0.25 & 88.62 \\
\hline $\mathrm{CGH}$ & 12 & 41.7320 & 18.1847 & 1140.00 & 140.00 & 242.00 & 0.12 & 0.21 & 47.61 \\
\hline $\mathrm{CGH}$ & 7 & 41.7659 & 17.9989 & 1445.00 & 220.00 & 302.00 & 0.15 & 0.21 & 120.20 \\
\hline $\mathrm{CGH}$ & $1 \mathrm{a}$ & 41.8538 & 17.8735 & 1262.00 & 60.00 & 175.00 & 0.05 & 0.14 & 25.00 \\
\hline $\mathrm{CGH}$ & 4 & 41.9290 & 17.8201 & 1069.00 & 80.00 & 259.00 & 0.07 & 0.24 & 23.92 \\
\hline $\mathrm{CGH}$ & $3 a$ & 41.9143 & 17.8855 & 1095.00 & 80.00 & 354.00 & 0.07 & 0.32 & 25.10 \\
\hline $\mathrm{CGH}$ & 22 & 41.5388 & 18.3865 & 931.00 & 40.00 & 232.00 & 0.04 & 0.25 & 9.07 \\
\hline $\mathrm{CGH}$ & 27 & 41.5733 & 18.3741 & 1407.00 & 200.00 & 202.00 & 0.14 & 0.14 & 103.60 \\
\hline $\mathrm{CGH}$ & $19 \mathrm{a}$ & 41.6141 & 18.3585 & 1115.00 & 80.00 & 207.00 & 0.07 & 0.19 & 26.02 \\
\hline $\mathrm{CGH}$ & 28 & 41.6481 & 18.3561 & 1100.00 & 80.00 & 159.00 & 0.07 & 0.14 & 25.33 \\
\hline $\mathrm{CGH}$ & $21 \mathrm{a}$ & 41.6284 & 18.4052 & 785.00 & 40.00 & 149.00 & 0.05 & 0.19 & 6.45 \\
\hline $\mathrm{CGH}$ & $21 b$ & 41.6241 & 18.3867 & 1016.00 & 60.00 & 104.00 & 0.06 & 0.10 & 16.21 \\
\hline $\mathrm{CGH}$ & 38 & 41.5100 & 18.4603 & 807.00 & 40.00 & 126.00 & 0.05 & 0.16 & 6.82 \\
\hline $\mathrm{CGH}$ & 39 & 41.5537 & 18.4991 & 501.00 & 40.00 & 63.00 & 0.08 & 0.13 & 2.63 \\
\hline $\mathrm{CGH}$ & 60 & 41.5427 & 18.4586 & 439.00 & 20.00 & 116.00 & 0.05 & 0.26 & 1.01 \\
\hline $\mathrm{CGH}$ & 36 & 41.5772 & 18.4601 & 1081.00 & 80.00 & 189.00 & 0.07 & 0.17 & 24.46 \\
\hline $\mathrm{CGH}$ & 35 & 41.5874 & 18.4355 & 1382.00 & 60.00 & 314.00 & 0.04 & 0.23 & 29.99 \\
\hline $\mathrm{CGH}$ & 37 & 41.5883 & 18.4228 & 1083.00 & 80.00 & 190.00 & 0.07 & 0.18 & 24.55 \\
\hline $\mathrm{CGH}$ & $21 b$ & 41.6241 & 18.3867 & 676.00 & 20.00 & 190.00 & 0.03 & 0.28 & 2.39 \\
\hline $\mathrm{CGH}$ & 44 & 41.5720 & 18.3546 & 898.00 & 80.00 & 216.00 & 0.09 & 0.24 & 16.88 \\
\hline $\mathrm{CGH}$ & 41 & 41.5837 & 18.3370 & 740.00 & 20.00 & 217.00 & 0.03 & 0.29 & 2.87 \\
\hline $\mathrm{CGH}$ & 42 & 41.5718 & 18.3208 & 539.00 & 20.00 & 147.00 & 0.04 & 0.27 & 1.52 \\
\hline $\mathrm{CGH}$ & 46 & 41.5332 & 18.2735 & 677.00 & 20.00 & 261.00 & 0.03 & 0.39 & 2.40 \\
\hline $\mathrm{CGH}$ & 45 & 41.5420 & 18.3074 & 696.00 & 60.00 & 160.00 & 0.09 & 0.23 & 7.61 \\
\hline $\mathrm{CGH}$ & 18 & 41.5569 & 18.2928 & 962.00 & 20.00 & 277.00 & 0.02 & 0.29 & 4.84 \\
\hline $\mathrm{CGH}$ & 43 & 41.5858 & 18.3090 & 1094.00 & 20.00 & 275.00 & 0.02 & 0.25 & 6.26 \\
\hline $\mathrm{CGH}$ & $26 \mathrm{a}$ & 41.5947 & 18.3189 & 820.00 & 20.00 & 244.00 & 0.02 & 0.30 & 3.52 \\
\hline $\mathrm{CGH}$ & $26 \mathrm{~b}$ & 41.6386 & 18.3025 & 1517.00 & 120.00 & 353.00 & 0.08 & 0.23 & 72.26 \\
\hline
\end{tabular}


Table 1. (Continued.)

\begin{tabular}{|c|c|c|c|c|c|c|c|c|c|}
\hline Zone & $\begin{array}{c}\text { Cone } \\
\text { no. }\end{array}$ & Long. & Lat. & $\begin{array}{r}\text { Cone_basal } \\
\left(W_{\mathrm{co}}\right)(\mathrm{m})\end{array}$ & $\begin{array}{c}\text { Cone_heigh } \\
\left(H_{\mathrm{co}}\right)(\mathrm{m})\end{array}$ & $\begin{array}{l}\text { Crater_dia } \\
\left(W_{\text {cr }}\right)(\mathrm{m})\end{array}$ & $H_{\mathrm{co}} / W_{\mathrm{co}}$ & $W_{\mathrm{cr}} / W_{\mathrm{co}}$ & $\begin{array}{l}\text { Volume } \\
\left(10^{6} \mathrm{~m}^{3}\right)\end{array}$ \\
\hline $\mathrm{CGH}$ & $19 \mathrm{c}$ & 41.6112 & 18.3320 & 887.00 & 80.00 & 116.00 & 0.09 & 0.13 & 16.47 \\
\hline $\mathrm{CGH}$ & 61 & 41.6240 & 18.2860 & 1088.00 & 80.00 & 139.00 & 0.07 & 0.13 & 24.78 \\
\hline $\mathrm{CGH}$ & $16 \mathrm{c}$ & 41.6164 & 18.2607 & 706.00 & 20.00 & 183.00 & 0.03 & 0.26 & 2.61 \\
\hline $\mathrm{CGH}$ & $16 \mathrm{~b}$ & 41.6289 & 18.2560 & 861.00 & 80.00 & 149.00 & 0.09 & 0.17 & 15.52 \\
\hline $\mathrm{CGH}$ & $16 \mathrm{a}$ & 41.6099 & 18.2400 & 705.00 & 40.00 & 159.00 & 0.06 & 0.23 & 5.20 \\
\hline $\mathrm{CGH}$ & 49 & 41.5972 & 18.2011 & 1506.00 & 140.00 & 222.00 & 0.09 & 0.15 & 83.09 \\
\hline $\mathrm{CGH}$ & $11 \mathrm{a}$ & 41.6154 & 18.1881 & 699.00 & 20.00 & 270.00 & 0.03 & 0.39 & 2.56 \\
\hline $\mathrm{CGH}$ & 50 & 41.5851 & 18.1626 & 845.00 & 40.00 & 183.00 & 0.05 & 0.22 & 7.47 \\
\hline $\mathrm{CGH}$ & $49 a$ & 41.5735 & 18.1923 & 1155.00 & 40.00 & 205.00 & 0.03 & 0.18 & 13.96 \\
\hline $\mathrm{CGH}$ & 48 & 41.5473 & 18.2245 & 601.00 & 20.00 & 144.00 & 0.03 & 0.24 & 1.89 \\
\hline $\mathrm{CGH}$ & 47 & 41.5494 & 18.2591 & 659.00 & 20.00 & 200.00 & 0.03 & 0.30 & 2.27 \\
\hline $\mathrm{CGH}$ & 59 & 41.6445 & 18.1305 & 667.00 & 60.00 & 155.00 & 0.09 & 0.23 & 6.98 \\
\hline $\mathrm{CGH}$ & $9 \mathrm{e}$ & 41.7108 & 18.0960 & 999.00 & 80.00 & 135.00 & 0.08 & 0.14 & 20.89 \\
\hline $\mathrm{CGH}$ & $9 \mathrm{~d}$ & 41.7579 & 18.0755 & 782.00 & 60.00 & 197.00 & 0.08 & 0.25 & 9.60 \\
\hline $\mathrm{CGH}$ & $8 \mathrm{a}$ & 41.7240 & 17.9974 & 1387.00 & 120.00 & 178.00 & 0.09 & 0.13 & 60.41 \\
\hline $\mathrm{CGH}$ & $8 b$ & 41.7341 & 17.9850 & 979.00 & 100.00 & 169.00 & 0.10 & 0.17 & 25.08 \\
\hline $\mathrm{CGH}$ & $8 c$ & 41.7407 & 17.9687 & 1025.00 & 80.00 & 189.00 & 0.08 & 0.18 & 21.99 \\
\hline $\mathrm{CGH}$ & $8 d$ & 41.7083 & 17.9738 & 748.00 & 60.00 & 152.00 & 0.08 & 0.20 & 8.78 \\
\hline $\mathrm{CGH}$ & 54 & 41.7219 & 17.9400 & 577.00 & 60.00 & 74.00 & 0.10 & 0.13 & 5.23 \\
\hline $\mathrm{CGH}$ & 62 & 41.7182 & 17.9549 & 899.00 & 60.00 & 79.00 & 0.07 & 0.09 & 12.69 \\
\hline $\mathrm{CGH}$ & 63 & 41.7741 & 17.8731 & 747.00 & 40.00 & 187.00 & 0.05 & 0.25 & 5.84 \\
\hline $\mathrm{CGH}$ & $12 \mathrm{c}$ & 41.7329 & 18.1672 & 1056.00 & 40.00 & 189.00 & 0.04 & 0.18 & 11.67 \\
\hline $\mathrm{CGH}$ & $2 \mathrm{c}$ & 41.8877 & 17.9593 & 735.00 & 80.00 & 194.00 & 0.11 & 0.26 & 11.31 \\
\hline $\mathrm{CGH}$ & 57 & 41.9422 & 17.8599 & 1436.00 & 40.00 & 335.00 & 0.03 & 0.23 & 21.58 \\
\hline $\mathrm{CGH}$ & $12 \mathrm{~b}$ & 41.7349 & 18.1527 & 658.00 & 40.00 & 176.00 & 0.06 & 0.27 & 4.53 \\
\hline $\mathrm{CGH}$ & $13 \mathrm{a}$ & 41.7586 & 18.2395 & 1613.00 & 180.00 & 330.00 & 0.11 & 0.20 & 122.54 \\
\hline $\mathrm{CGH}$ & $13 \mathrm{~b}$ & 41.7433 & 18.2310 & 843.00 & 80.00 & 168.00 & 0.09 & 0.20 & 14.88 \\
\hline $\mathrm{CGH}$ & 52 & 41.7814 & 18.2614 & 1059.00 & 120.00 & 243.00 & 0.11 & 0.23 & 35.21 \\
\hline $\mathrm{CGH}$ & 65 & 18.2614 & 18.3094 & 1363.00 & 160.00 & 234.00 & 0.12 & 0.17 & 77.78 \\
\hline $\mathrm{CGH}$ & 66 & 41.7209 & 18.3293 & 950.00 & 160.00 & 245.00 & 0.17 & 0.26 & 37.78 \\
\hline $\mathrm{CGH}$ & $20 \mathrm{a}$ & 41.6991 & 18.3732 & 974.00 & 120.00 & 203.00 & 0.12 & 0.21 & 29.79 \\
\hline $\mathrm{CGH}$ & 29 & 41.6829 & 18.4258 & 1085.00 & 100.00 & 173.00 & 0.09 & 0.16 & 30.80 \\
\hline $\mathrm{CGH}$ & $29 a$ & 41.6826 & 18.4498 & 862.00 & 20.00 & 207.00 & 0.02 & 0.24 & 3.89 \\
\hline $\mathrm{CGH}$ & 30 & 41.7037 & 18.4260 & 943.00 & 100.00 & 146.00 & 0.11 & 0.15 & 23.27 \\
\hline $\mathrm{CGH}$ & 31 & 41.7147 & 18.4389 & 1198.00 & 20.00 & 171.00 & 0.02 & 0.14 & 7.51 \\
\hline $\mathrm{CGH}$ & $31 \mathrm{a}$ & 41.7132 & 18.4519 & 900.00 & 40.00 & 287.00 & 0.04 & 0.32 & 8.48 \\
\hline $\mathrm{CGH}$ & 32 & 41.7149 & 18.4662 & 881.00 & 40.00 & 231.00 & 0.05 & 0.26 & 8.12 \\
\hline $\mathrm{CGH}$ & 34 & 41.7325 & 18.4799 & 1926.00 & 160.00 & 190.00 & 0.08 & 0.10 & 155.30 \\
\hline $\mathrm{CGH}$ & 33 & 41.7247 & 18.4968 & 1169.00 & 200.00 & 253.00 & 0.17 & 0.22 & 71.52 \\
\hline $\mathrm{CGH}$ & 58 & 41.6603 & 18.0397 & 1292.00 & 20.00 & 133.00 & 0.02 & 0.10 & 8.74 \\
\hline $\mathrm{CGH}$ & 53 & 41.6542 & 18.0078 & 1049.00 & 100.00 & 103.00 & 0.10 & 0.10 & 28.79 \\
\hline $\mathrm{CGH}$ & 67 & 41.6938 & 17.8936 & 508.00 & 20.00 & 112.00 & 0.04 & 0.22 & 1.35 \\
\hline $\mathrm{CGH}$ & 68 & 41.8369 & 17.9514 & 882.00 & 80.00 & 165.00 & 0.09 & 0.19 & 16.28 \\
\hline $\mathrm{CGH}$ & 69 & 41.6617 & 18.2758 & 643.00 & 100.00 & 77.00 & 0.16 & 0.12 & 10.82 \\
\hline $\mathrm{CGH}$ & $15 \mathrm{a}$ & 41.6602 & 18.2876 & 923.00 & 80.00 & 286.00 & 0.09 & 0.31 & 17.83 \\
\hline $\mathrm{CGH}$ & 70 & 41.6348 & 18.2850 & 791.00 & 40.00 & 199.00 & 0.05 & 0.25 & 6.55 \\
\hline $\mathrm{CGH}$ & 71 & 41.5862 & 18.2962 & 585.00 & 40.00 & 186.00 & 0.07 & 0.32 & 3.58 \\
\hline $\mathrm{CGH}$ & 72 & 41.7270 & 18.2029 & 625.00 & 60.00 & 97.00 & 0.10 & 0.16 & 6.13 \\
\hline $\mathrm{CGH}$ & 51 & 41.7963 & 18.2420 & 705.00 & 100.00 & 117.00 & 0.14 & 0.17 & 13.01 \\
\hline $\mathrm{CGH}$ & $19 \mathrm{~b}$ & 41.6223 & 18.3311 & 730.00 & 60.00 & 247.00 & 0.08 & 0.34 & 8.37 \\
\hline $\mathrm{CGH}$ & $19 \mathrm{~d}$ & 41.6285 & 18.3374 & 757.00 & 40.00 & 233.00 & 0.05 & 0.31 & 6.00 \\
\hline
\end{tabular}


Table 1. (Continued.)

\begin{tabular}{|c|c|c|c|c|c|c|c|c|c|}
\hline Zone & $\begin{array}{c}\text { Cone } \\
\text { no. }\end{array}$ & Long. & Lat. & $\begin{array}{r}\text { Cone_basal } \\
\left(W_{\mathrm{co}}\right)(\mathrm{m})\end{array}$ & $\begin{array}{c}\text { Cone_heigh } \\
\left(H_{\mathrm{co}}\right)(\mathrm{m})\end{array}$ & $\begin{array}{l}\text { Crater_dia } \\
\left(W_{\mathrm{cr}}\right)(\mathrm{m}) \\
\end{array}$ & $H_{\mathrm{co}} / W_{\mathrm{co}}$ & $W_{\mathrm{cr}} / W_{\mathrm{co}}$ & $\begin{array}{l}\text { Volume } \\
\left(10^{6} \mathrm{~m}^{3}\right)\end{array}$ \\
\hline $\mathrm{CGH}$ & 40 & 41.5419 & 18.4890 & 585.00 & 60.00 & 219.00 & 0.10 & 0.37 & 5.37 \\
\hline $\mathrm{CGH}$ & $13 \mathrm{c}$ & 41.7321 & 18.2613 & 553.00 & 40.00 & 154.00 & 0.07 & 0.28 & 3.20 \\
\hline $\mathrm{CGH}$ & $13 \mathrm{~d}$ & 41.7498 & 18.2594 & 1430.00 & 80.00 & 168.00 & 0.06 & 0.12 & 42.81 \\
\hline CGH & $17 \mathrm{a}$ & 41.6452 & 18.2410 & 588.00 & 60.00 & 169.00 & 0.10 & 0.29 & 5.43 \\
\hline $\mathrm{CGH}$ & 56 & 41.6781 & 18.3244 & 603.00 & 20.00 & 180.00 & 0.03 & 0.30 & 1.90 \\
\hline $\mathrm{CGH}$ & 55 & 41.8451 & 17.8201 & 1462.00 & 120.00 & 385.00 & 0.08 & 0.26 & 67.12 \\
\hline $\mathrm{CGH}$ & 64 & 41.7186 & 18.0217 & 946.00 & 60.00 & 217.00 & 0.06 & 0.23 & 14.05 \\
\hline $\mathrm{CGH}$ & 77 & 41.6916 & 18.5105 & 512.00 & 20.00 & 152.00 & 0.04 & 0.30 & 1.37 \\
\hline $\mathrm{CGH}$ & $81 c$ & 41.7092 & 18.5655 & 871.00 & 60.00 & 254.00 & 0.07 & 0.29 & 11.91 \\
\hline $\mathrm{CGH}$ & 82 & 41.6577 & 18.5608 & 851.00 & 60.00 & 246.00 & 0.07 & 0.29 & 11.37 \\
\hline $\mathrm{CGH}$ & 83 & 41.6310 & 18.5271 & 845.00 & 40.00 & 174.00 & 0.05 & 0.21 & 7.47 \\
\hline $\mathrm{CGH}$ & 84 & 41.6045 & 18.5429 & 913.00 & 40.00 & 238.00 & 0.04 & 0.26 & 8.72 \\
\hline $\mathrm{CGH}$ & 85 & 41.7145 & 18.5223 & 651.00 & 100.00 & 183.00 & 0.15 & 0.28 & 11.09 \\
\hline $\mathrm{CGH}$ & 86 & 41.7124 & 18.6056 & 1124.00 & 80.00 & 246.00 & 0.07 & 0.22 & 26.45 \\
\hline $\mathrm{CGH}$ & 87 & 41.6044 & 18.5672 & 975.00 & 60.00 & 274.00 & 0.06 & 0.28 & 14.92 \\
\hline $\mathrm{CGH}$ & 88 & 41.6178 & 18.5777 & 1377.00 & 40.00 & 376.00 & 0.03 & 0.27 & 19.85 \\
\hline $\mathrm{CGH}$ & 89 & 41.6181 & 18.5607 & 525.00 & 60.00 & 131.00 & 0.11 & 0.25 & 4.33 \\
\hline $\mathrm{CGH}$ & 90 & 41.6370 & 18.5494 & 1050.00 & 80.00 & 260.00 & 0.08 & 0.25 & 23.08 \\
\hline $\mathrm{CGH}$ & 91 & 41.6254 & 18.6197 & 1440.00 & 120.00 & 343.00 & 0.08 & 0.24 & 65.11 \\
\hline $\mathrm{CGH}$ & 92 & 41.6781 & 18.6426 & 753.00 & 20.00 & 140.00 & 0.03 & 0.19 & 2.97 \\
\hline $\mathrm{CGH}$ & $92 \mathrm{a}$ & 41.6717 & 18.6579 & 976.00 & 120.00 & 153.00 & 0.12 & 0.16 & 29.91 \\
\hline $\mathrm{CGH}$ & 93 & 41.6210 & 18.6912 & 553.00 & 40.00 & 194.00 & 0.07 & 0.35 & 3.20 \\
\hline $\mathrm{CGH}$ & 94 & 41.5568 & 18.5469 & 1047.00 & 80.00 & 256.00 & 0.08 & 0.24 & 22.95 \\
\hline $\mathrm{CGH}$ & 95 & 41.5215 & 18.5652 & 635.00 & 40.00 & 242.00 & 0.06 & 0.38 & 4.22 \\
\hline $\mathrm{CGH}$ & $95 \mathrm{a}$ & 41.5359 & 18.5935 & 628.00 & 80.00 & 126.00 & 0.13 & 0.20 & 8.26 \\
\hline $\mathrm{CGH}$ & 96 & 41.5607 & 18.6700 & 401.00 & 20.00 & 94.00 & 0.05 & 0.23 & 0.84 \\
\hline $\mathrm{CGH}$ & 97 & 41.6109 & 18.7347 & 797.00 & 40.00 & 195.00 & 0.05 & 0.24 & 6.65 \\
\hline $\mathrm{CGH}$ & 99 & 41.7340 & 18.1444 & 546.00 & 40.00 & 126.00 & 0.07 & 0.23 & 3.12 \\
\hline $\mathrm{CGH}$ & $12 \mathrm{a}$ & 41.7540 & 18.1794 & 1019.00 & 200.00 & 355.00 & 0.20 & 0.35 & 54.34 \\
\hline $\mathrm{CGH}$ & 98 & 41.7337 & 18.4690 & 735.00 & 20.00 & 231.00 & 0.03 & 0.31 & 2.83 \\
\hline $\mathrm{CGH}$ & $1 b$ & 41.8430 & 17.9023 & 2277.00 & 200.00 & 774.00 & 0.09 & 0.34 & 271.33 \\
\hline $\mathrm{CGH}$ & $19 \mathrm{e}$ & 41.6316 & 18.3724 & 432.00 & 10.00 & 102.00 & 0.02 & 0.24 & 0.49 \\
\hline AGL & 73 & 42.0988 & 18.0185 & 1160.00 & 140.00 & 366.00 & 0.12 & 0.32 & 49.29 \\
\hline AGL & 74 & 42.3589 & 17.7083 & 660.00 & 40.00 & 200.00 & 0.06 & 0.30 & 4.56 \\
\hline AGL & 75 & 41.8367 & 18.3036 & 733.00 & 60.00 & 173.00 & 0.08 & 0.24 & 8.44 \\
\hline AGL & 76 & 41.8597 & 18.3738 & 1044.00 & 60.00 & 211.00 & 0.06 & 0.20 & 17.11 \\
\hline AGL & 78 & 41.7945 & 18.5111 & 522.00 & 20.00 & 94.00 & 0.04 & 0.18 & 1.43 \\
\hline AGL & 79 & 41.7836 & 18.5551 & 633.00 & 80.00 & 181.00 & 0.13 & 0.29 & 8.39 \\
\hline AGL & 80 & 41.7823 & 18.5429 & 746.00 & 40.00 & 217.00 & 0.05 & 0.29 & 5.82 \\
\hline AGL & $81 a$ & 41.8308 & 18.5571 & 451.00 & 60.00 & 153.00 & 0.13 & 0.34 & 3.19 \\
\hline AGL & $81 b$ & 41.8140 & 18.5967 & 494.00 & 100.00 & 179.00 & 0.20 & 0.36 & 6.39 \\
\hline AGL & 98 & 42.0594 & 18.5163 & 1977.00 & 160.00 & 428.00 & 0.08 & 0.22 & 163.64 \\
\hline JSGH & $97 \mathrm{a}$ & 42.9121 & 18.4003 & 1190.00 & 60.00 & 255.00 & 0.05 & 0.21 & 22.23 \\
\hline JSGH & 100 & 42.6079 & 18.1315 & 9606.00 & 1220.00 & 3706.00 & 0.13 & 0.39 & $29,457.33$ \\
\hline JSGH & $100 \mathrm{a}$ & 42.4310 & 18.1502 & 5991.00 & 780.00 & 1586.00 & 0.13 & 0.26 & 7325.57 \\
\hline JSGH & $100 \mathrm{~b}$ & 42.3420 & 18.2530 & 1698.00 & 520.00 & 502.00 & 0.31 & 0.30 & 392.31 \\
\hline JSGH & $100 \mathrm{c}$ & 42.3005 & 18.3527 & 1410.00 & 220.00 & 350.00 & 0.16 & 0.25 & 114.45 \\
\hline JSGH & $100 \mathrm{~d}$ & 42.4733 & 18.1806 & 2207.00 & 80.00 & 504.00 & 0.04 & 0.23 & 101.96 \\
\hline
\end{tabular}

CGH: coastal gravity high, AGL: axial gravity low, JSGH: Jabal As-Sawda gravity high (see figure 10 for illustration). 


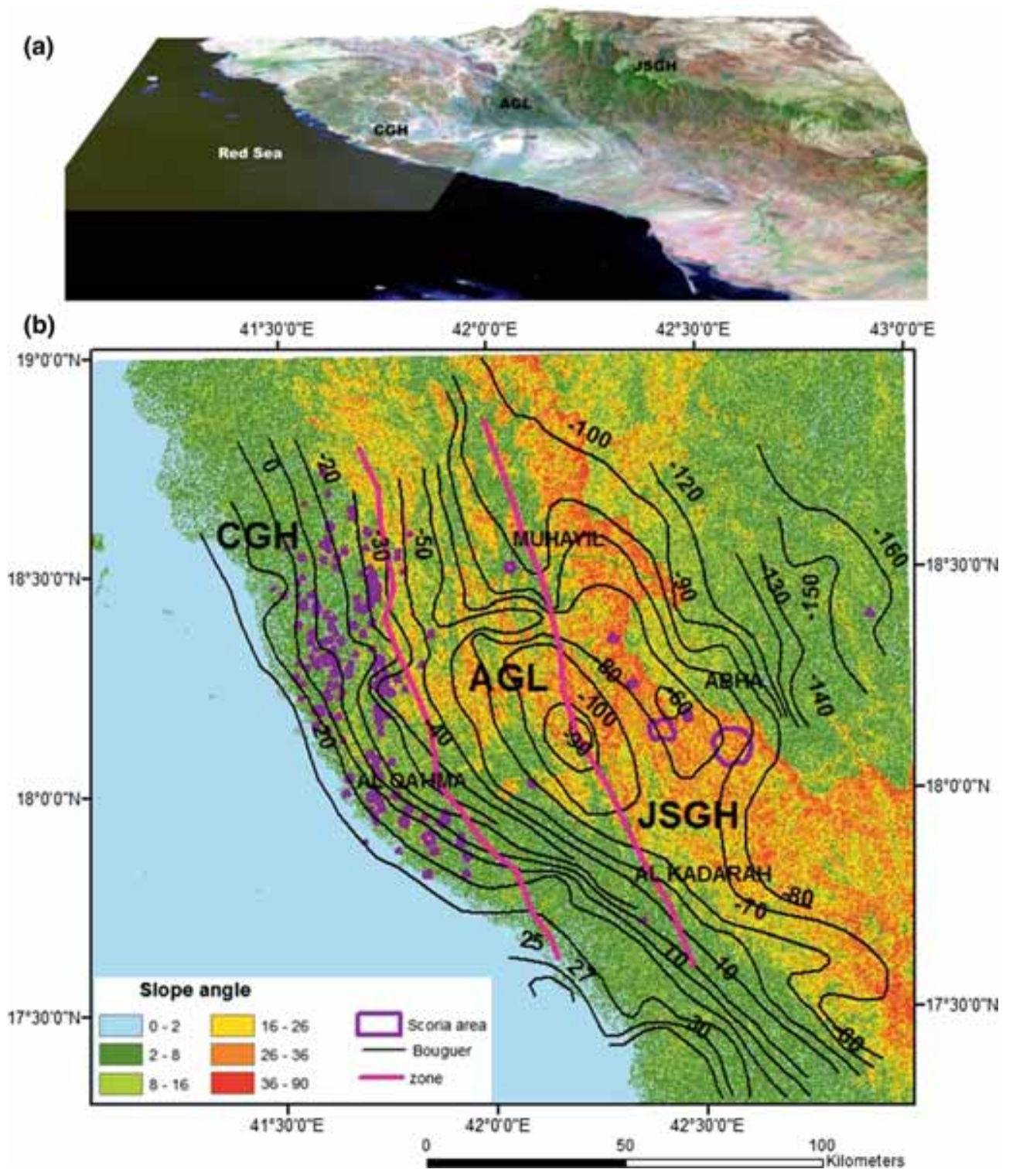

Figure 6. Satellite image analysis for cinder cones in the HAB. (a) Topography in the 3D model and (b) slope maps with slope degrees illustrated by different colour representations. The Bouguer gravity contours are draped with the demarcation of zones such as CGH: coastal gravity high, AGL: axial gravity low, JSGH: Jabal As-Sawda gravity high.

lava flows in $\mathrm{HAB}$ erupted from these cinder cones even though their individual volume of eruption may not be very large. The HAB cinder cones are now deeply dissected, but originally most of them are believed to have been at least $150 \mathrm{~m}$ high and $800-1500 \mathrm{~m}$ in base diameter (Coleman et al. 1983). Xenoliths are common in the cinder cones and so also within the solidified flows; Ghent et al. (1980) consider this to reflect the lower crustal composition beneath HAB. Here, we estimate the morphological characteristics of the larger cinder cones using satellite images (figure 7 ). Where the $\mathrm{HAB}$ volcanic rocks are exposed by erosion, as in the Wadi Amq and Wadi Dhahaban, the flows are columnar in the lower half and grade upward to a zone of knobby rock that is overlain by a zone of platy, horizontally fractured rock. This has given an impression that the lavas must have been very fluid and most of the volume might have flowed towards the Red Sea. The average geomorphic slope in CGH is very low $\left(2^{\circ}-8^{\circ}\right)$ towards the Red Sea. The topographic profiles across the Jabal at Tusi Ash Shami (No. 1), Jabal at Tusi Al-Yamani (No. 2), Jabal Qurayn (No. 3) and Al-Birk Cinder (No. 4) show prominent cone structures with rather steep slopes on both sides (figure 7). 

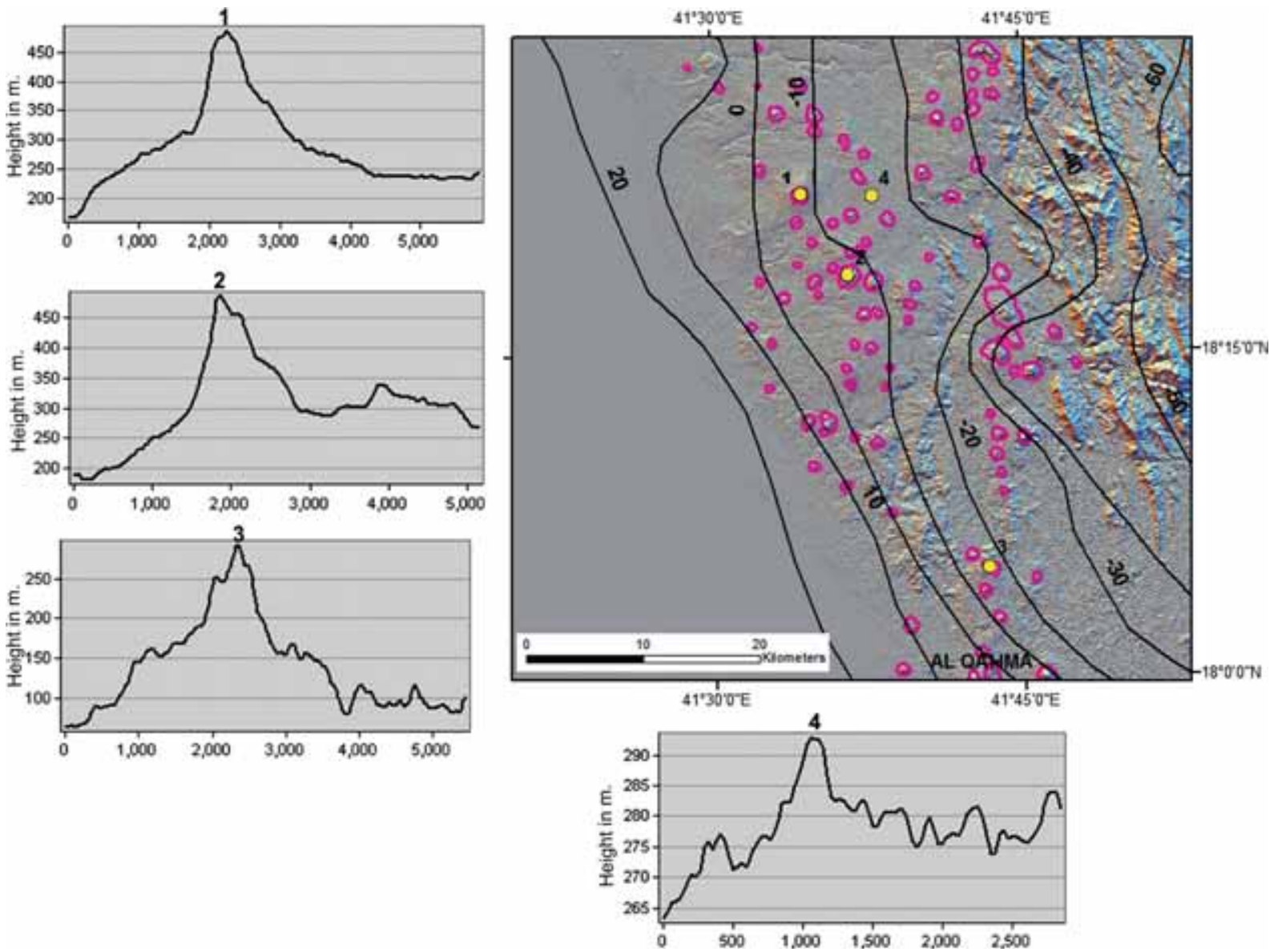

Figure 7. Satellite image analysis for cinder cones in the HAB in the zone of the CGH with gravity anomalies contours. The prominent cinder cones are marked as Jabal at Tusi Ash Shami (No. 1), Jabal at Tusi Al-Yamani (No. 2), Jabal Qurayn (No. 3) and Al-Birk Cinder (No. 4). The profiles across the cinder cones are marked with height in metres.

\subsection{Cinder cones in the Asir foreland corresponding to the $A G L$}

Prominent cinders located in AGL are the Jabal Al-Haylah (No. 1, elevation $710 \mathrm{~m}$ ) that occur as an isolated cinder, Jabal Ba'a (No. 2) and Jabal Al-Qishr (No. 3, elevation $362 \mathrm{~m}$ which also occurs as an isolated cinders). Here the average geomorphic slope is low, ranging between $8^{\circ}$ and $16^{\circ}$. The topographic profiles across the cinders show both prominent and denuded cone structures with moderate to gentle slopes on both sides (figure 8).

\subsection{Cinder cones situated over the foreland of the Asir mountain - zone of JSGH}

The cinder cones in the foreland of the Asir mountains located west of the tourist town Abha $\left(18^{\circ} 13^{\prime} 0.47^{\prime \prime} \mathrm{N} ; \quad 42^{\circ} 30^{\prime} 13.55^{\prime \prime} \mathrm{E}\right)$ are listed below together with their elevations (h in metres): Jabal As-Sawda (No. 1, h 3015 m), Jabal As-Seqaa
(No. 2, h 2863 m), Jabal Nahran (No. 3, h 2837 m), Jabal Shaokan (No. 4, h 2786 m), Jabal Rathba (No. 5, h $2744 \mathrm{~m}$ ) and Jabal Al-Jabha'a (No. 6, h $2581 \mathrm{~m})$.

All six cinders are conspicuously developed within the JSGH, the Jabal As-Sawda forming the highest cliff in the As-Sarawat mountains (figure 9). The average geomorphic slope in JSGH is in between $16^{\circ}$ and $36^{\circ}$, but at some places, subvertical slopes forming scarp are also observed. The topographic profiles across these cinders illustrate the disposition of the highly denuded cone structures with an abrupt change in slope (figure 9).

\subsection{Morphometric parameters and analysis of the cinder cones of CGH, AGL and JSGH}

Morphometric measurements: $W_{\mathrm{co}}, H_{\mathrm{co}}, W_{\mathrm{cr}}$, cone slope (for illustration of cone disposition in plan and section, and corresponding measurements, refer figure 10) and cone volume and their aspect ratios 

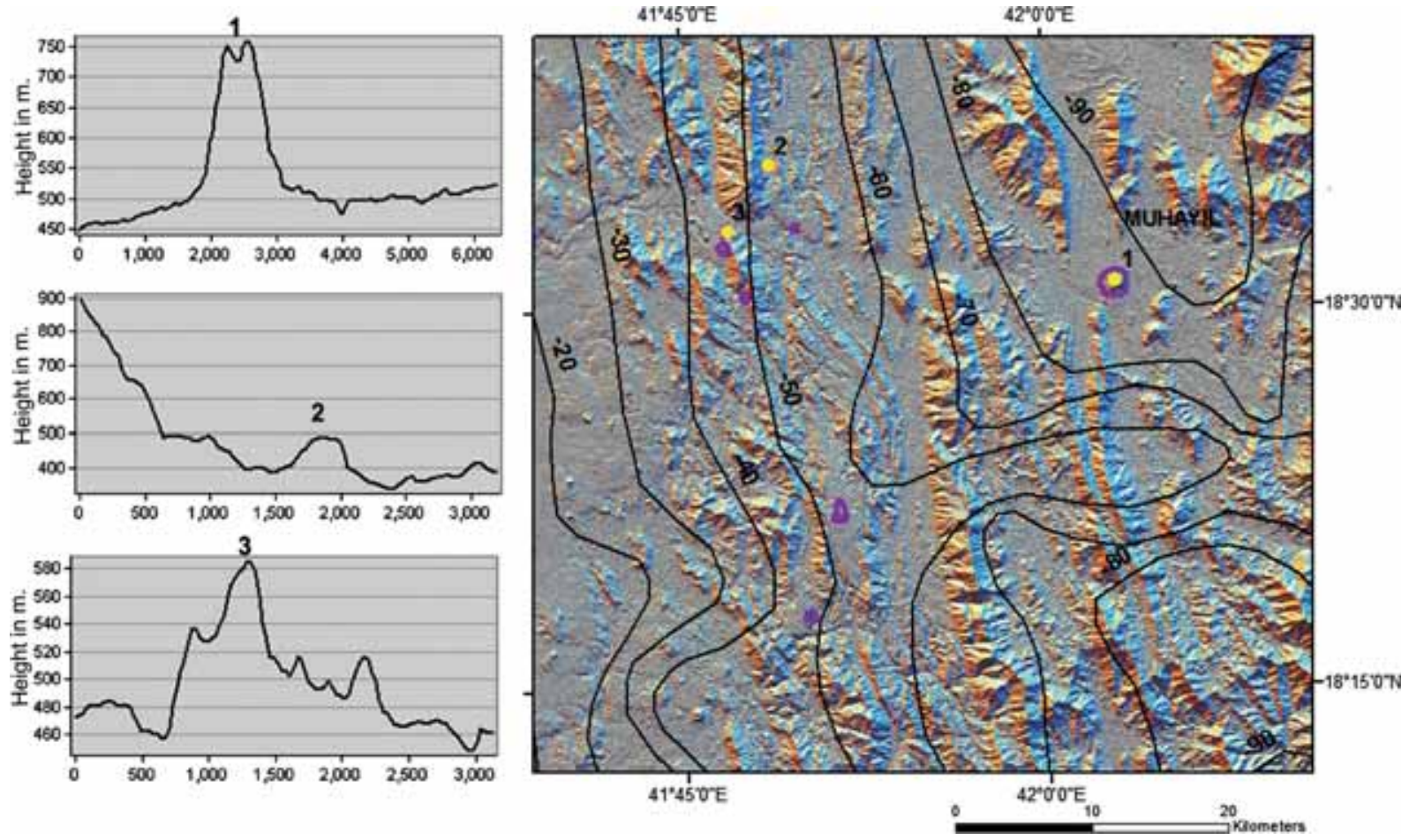

Figure 8. Satellite image analysis for cinder cones in the HAB in the zone of the AGL with gravity anomalies contours. The prominent cinder cones are marked as Jabal Al-Haylah (No. 1), JabalBa'a (No. 2) and Jabal Al-Qishr (No. 3). The profiles across the cinder cones are marked with height in metres.

$\left(H_{\mathrm{co}} / W_{\mathrm{co}}\right.$ and $\left.W_{\mathrm{cr}} / W_{\mathrm{co}}\right)$ for the 150 cinder cones belonging to CGH, AGL and JSGH are given in table 1 and their relationships are illustrated in figure 11. Data listed here are: the average cone height, cone diameter, crater diameter and cone volume, while their relationship involving the cone height and the cone basal diameter is illustrated in figure $11(\mathrm{a}, \mathrm{d}$ and $\mathrm{g}$ ) for $\mathrm{CGH}$, AGL and JSGH, respectively. Major observations resulting from these plots are the great variability in morphometric ratios, even for cones which are of simple shape and well preserved. The cone height is 0.07 times the cone basal diameter for $\mathrm{CGH}$, and 0.08 for AGL and that for JSGH is 0.12 . The plot between the crater diameter and the cone basal diameter shows positive correlation. The crater diameter is 0.19 times the cone basal diameter for CGH, 0.15 times for AGL and 0.2 times for JSGH. The $H_{\mathrm{co}} / W_{\mathrm{co}}$ against the volume plot shows a positive correlation for $\mathrm{CGH}$, AGL and JSGH (figure 11b, e and $\mathrm{h}$ ). The analysis gives an overall view of the relationship between the $H_{\mathrm{co}} / W_{\mathrm{co}}$ and cone volume which is suggestive of a broad envelope for the lifespan of the scoria for CGH. Normally, the degradation of cinder cones with age should result in a decrease in cone volume with a corresponding decrease in the $H_{\mathrm{co}} / W_{\text {co }}$ ratio. The plots of $H_{\mathrm{co}} / W_{\mathrm{co}}$ against volume show a similar trend for CGH, AGL and JSGH. Often, the ratio of $H_{\mathrm{co}} / W_{\mathrm{co}}$ is largely affected by the rate of erosion; erosion lowers the ratio of $H_{\mathrm{co}} / W_{\mathrm{co}}$. Cinder cones in the semi-arid regions of the world have shown as low an $H_{\text {co }} / W_{\text {co }}$ ratio as 0.08 (Hooper and Sheridan 1998). Similarly, JSGH is the oldest amongst the three volcanic zones; it is characterised by a high $H_{\mathrm{co}} / W_{\text {co }}$ ratio. Large cinder cones of JSGH are characterised by angular asymmetry at their base angles. The plot of $W_{\text {co }} v s$. $W_{\text {cr }}$ for all gravity zones indicates very low valued positive correlation (figure 11c, f and i).

Morphometric parameters, in particular, the slope angle can provide valuable information about the age of scoria edifices or cones. Following the model given by Wood (1980a,b), the cone's initial slopes range from $30^{\circ}$ to $33^{\circ}$ measured either manually or from topographic maps, assuming that the inner architectures of the cones are homogeneous. In the JSGH area, the average slope is $27^{\circ}-40^{\circ}$, whereas in AGL, it is $25^{\circ}$ and for the $\mathrm{CGH}$ area, it is $2^{\circ}-15^{\circ}$ (see figure 5 ). The $H_{\mathrm{co}} / W_{\text {co }}$ ratio is 0.02 times the volume for JSGH, whereas this is comparatively very low (0.0008) for CGH and 0.01 times for AGL. In general, a higher $H_{\mathrm{co}} / W_{\text {co }}$ ratio indicates a steeper slope angle and thus the 

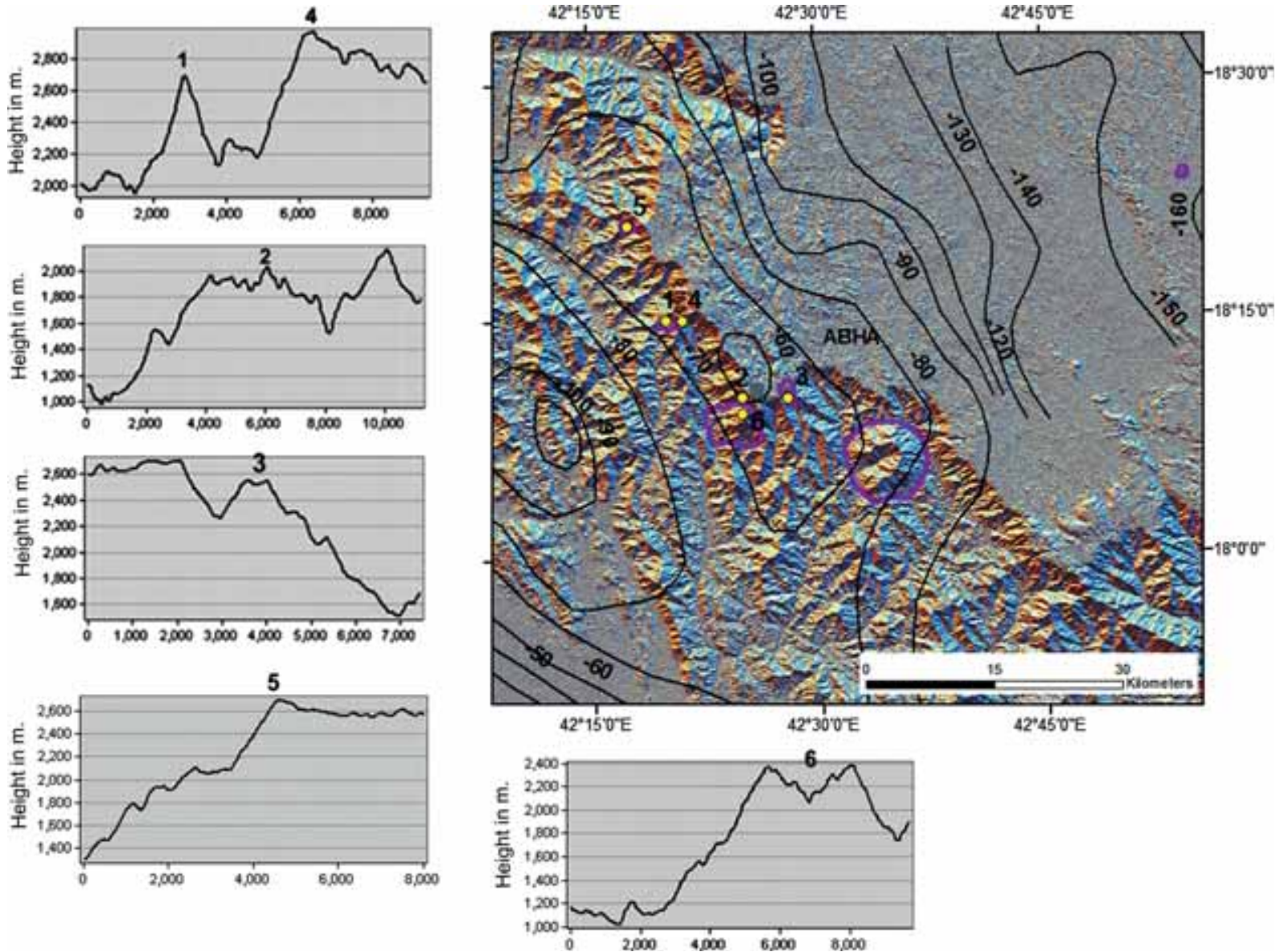

Figure 9. Satellite image analysis for cinder cones in the As-Sarawat mountains - zone of the JSGH with gravity anomaly contours. The prominent cinder cones are marked as Jabal As-Sawda (No. 1), Jabal As-Seqaa (No. 2), Jabal Nahran (No. 3 ), Jabal Shaokan (No. 4), Jabal Rathba (No. 5) and Jabal Al-Jabha'a (No. 6). The profiles across the cinder cones are marked with height in metres.
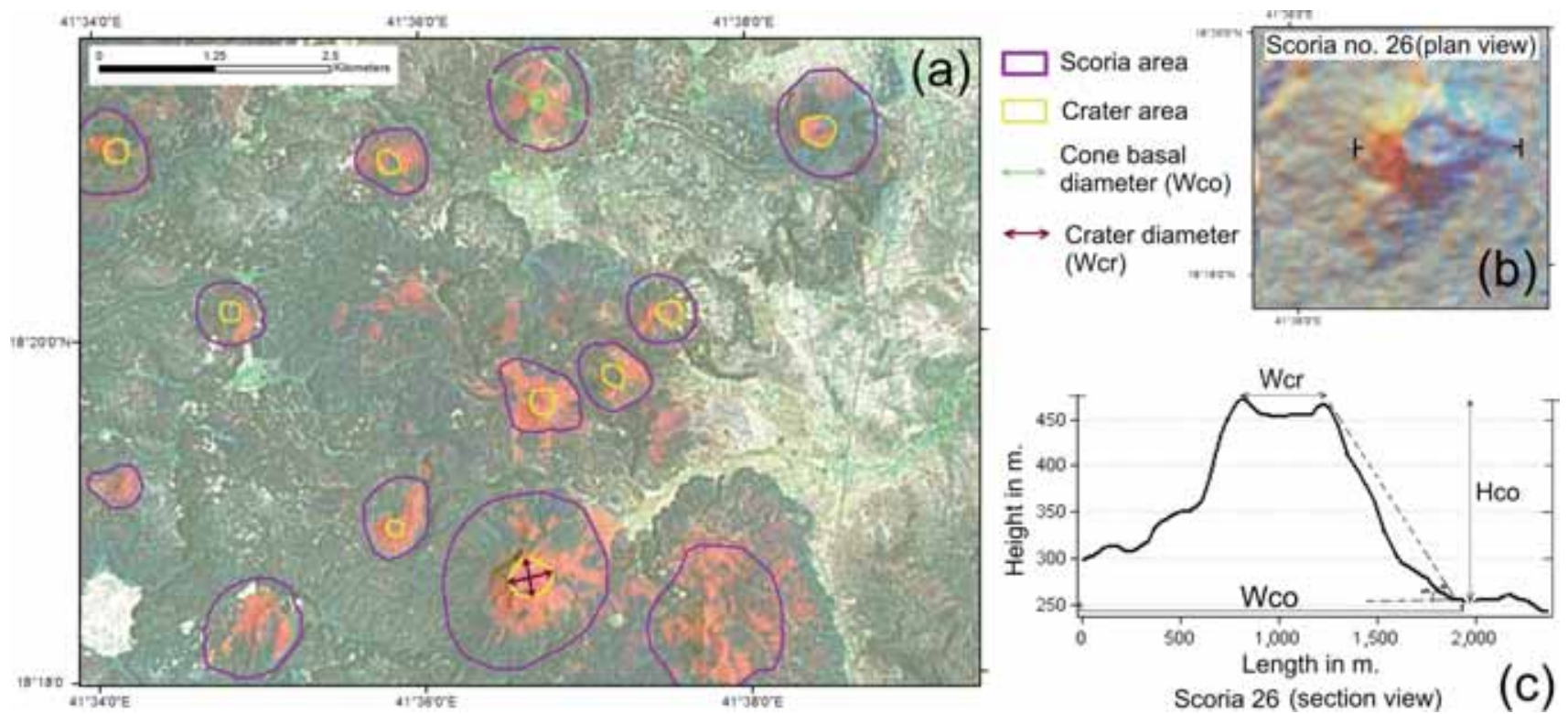

Figure 10. Illustration for the morphometric measurements done on the cinder cones. (a) Part of the satellite imagery showing juvenile cinder cones belonging to the gravity zone CGH, where the scoria area, crater area, cone basal diameter $\left(W_{\text {co }}\right)$ and crater diameter $\left(W_{\text {cr }}\right)$ are marked with symbols. (b) One of the prominent scoria cones (cone no. 26 of table 1 ) is shown in plan-view and cross section (c) with measured parameters $W_{\mathrm{co}}, W_{\mathrm{cr}}$, cone height $\left(H_{\mathrm{co}}\right)$ and cone slope $(\alpha)$. 


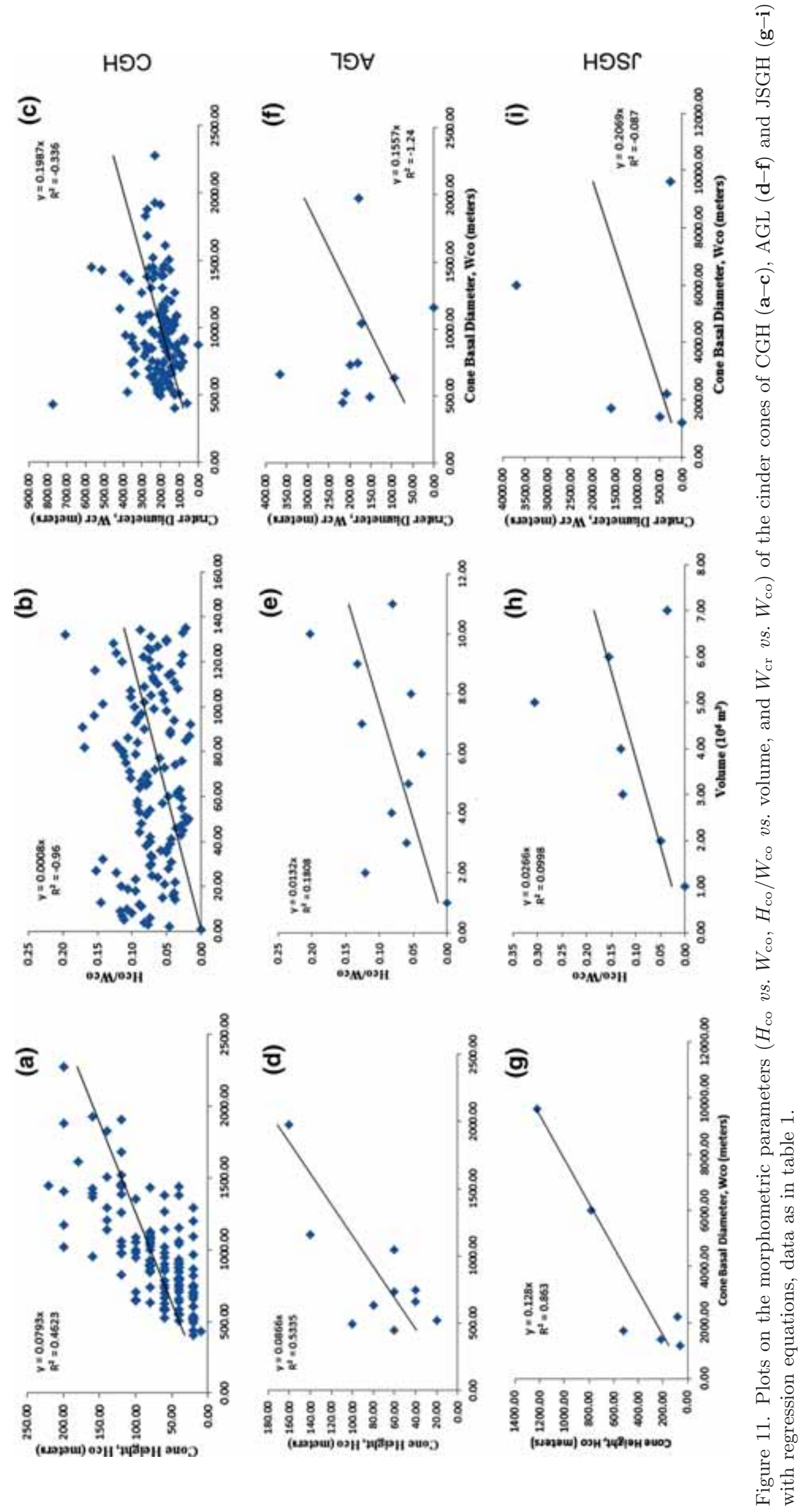


(a)

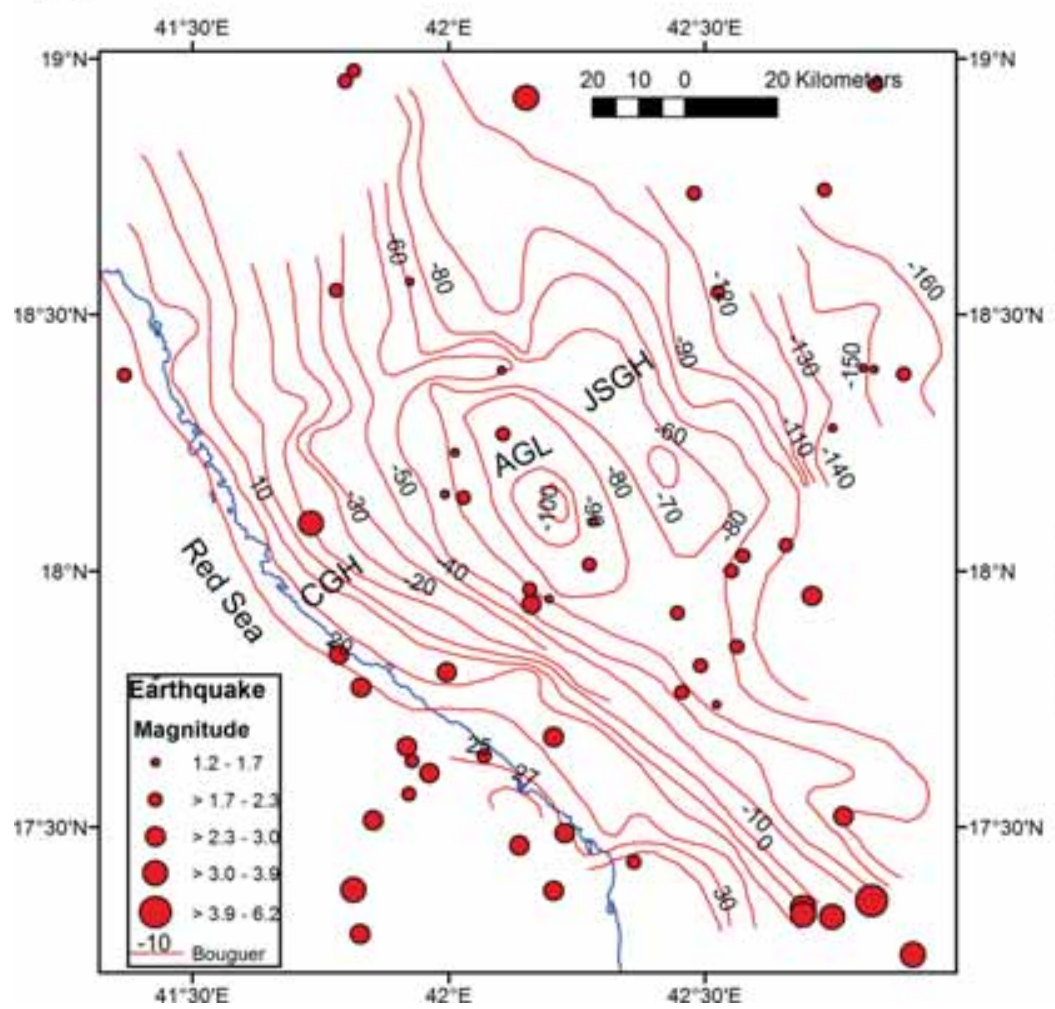

(b)

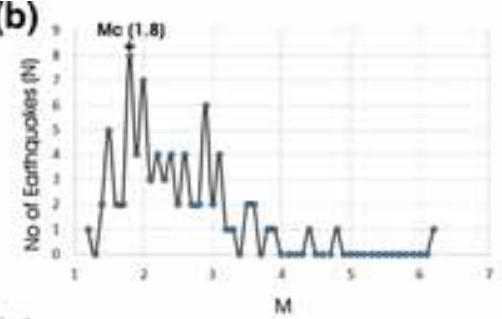

(c)

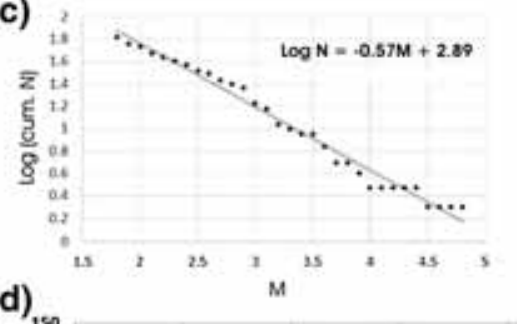

(d)

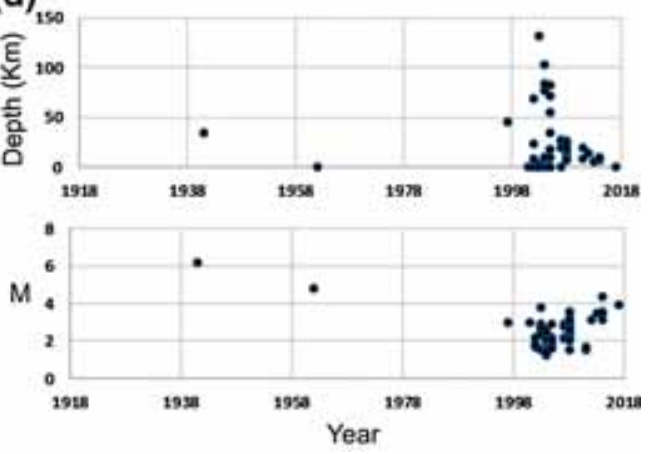

Figure 12. Map showing earthquake locations with magnitude in the HAB and Asir foreland. They are mostly of crustal origin and generated in response to extensional tectonism prevailing in the area. (a) Map showing disposition of earthquake occurrences with magnitude $(M)$ as size bin; (b) magnitude $(M)$ vs. frequency plot $(N)$ to delineate magnitude completeness $\left(M_{c}\right)$ as highest frequency, $M_{c}$ is $1.8 ;(\mathbf{c})$ calculated $b$-value by $\mathrm{G}-\mathrm{R}$ regression method, $b=0.57, a=2.89$, and $(\mathbf{d})$ plot of occurrence year vs. magnitude and focal depth of the earthquakes.

morphological slope for JSGH is steeper compared to the slope along AGL and CGH. However, the high slope values observed along the eastern part of JSGH with steep river valleys are formed as a result of the rejuvenation of the streams which were earlier occupied by the lava flows. The steep slope is also indicative of high erosive action by the river in the lava fields.

It is known that morphometric variability can be influenced by factors such as pre-eruptive surface inclination, vent migration and lava outflow with associated crater breaching and the diversity of pyroclastic rocks that are accumulated in the flanks of these volcanic edifices (Kereszturi et al. 2012). We conjecture that the two morphometric ratios $\left(W_{\mathrm{cr}} / W_{\mathrm{co}}\right.$ and $\left.H_{\mathrm{co}} / W_{\mathrm{co}}\right)$, their average values and their repose angle of loose granular scoria control the cone slopes. The observed variability in the ratios between individual cones is probably an outcome of erosion process and/or contrasting eruptive conditions. Furthermore, the erosion vs. time relationships will be skewed if naturally formed cinder cones are of different materials (Kereszturi and Németh 2012a,b). The traditional formula-based morphometric parameterisation may not always strictly correlate with the age of the cinder cones (Kereszturi and Németh 2012a, b); instead, the DEM-based slope angle estimation method is found to be more precise and realistic when compared with the radiometric ages of the cinder cones. To some extent, this argument holds good for the present case dealing with the older cones in JSGH to the younger cones in CGH.

\subsection{Earthquake data in the $H A B$ and Asir foreland in relation to volcanism}

The tele-seismic earthquake data for the period 1941-2017 shows 78 occurrences (figure 12) with magnitude ranging from 1.2 to 6.2 as per ISC catalogue in the area. The concentration of earthquake is seen between 1998 and 2017 with a magnitude below 4.0, mainly concentrated in the CGH zone 
(figure 12a) which are distinctly of crustal origin (figure $12 \mathrm{~d}$ ). The data having magnitude completeness $\left(M_{\mathrm{c}} \geq 1.8\right.$; figure $\left.12 \mathrm{~b}\right)$ shows a $b$ value of $0.52 \pm 0.06$ (as per the likelihood method with a $b$-value error; Aki 1965) and $0.57 \pm 0.05$ (as per the $\mathrm{G}-\mathrm{R}$ regression method; figure $12 \mathrm{c}$ ). The low $b$ value connotes for their extensional tectonic origin rather than of pure volcanic origin.

\section{Lineament analysis of cinder cones based on satellite data}

Lineaments are the linear structures commonly associated with tectonic deformation showing dislocation. A lineament is a linear or curvilinear feature in a regional scale, its pattern or change in pattern may be attributed to analysing the structure and tectonic aspects of the study area. Larger lineaments have a large zone of influence and may represent a larger amount of deformation associated with them and the present study may epitomise the feeder sub-system within an overall volcanic system. It is known that remote sensing images and associated enhancement techniques are the best methods to identify linear features like faults and fracture systems that may act as conduits for mobilisation of volcanic material. Accordingly, a lineament map of the study area was generated using freely available Landsat ETM+ (colour ETM+PAN sharpened ortho product) data along with a shaded relief map of the study area. The scenes of path rows 167_47 and 167_48 were mosaic using the ENVI 4.8 software, and subsequently, the area was subset using latitude-longitude by the GIS software. The subset image was spectrally stretched and enhanced to get the best possible image for visual interpretation and the subsequent demarcation of lineaments.

Based on image interpretation, most of the lineaments follow the trend of the major shear zone, fault, fracture and dyke systems of the study area (figure 13). Some lineaments are parallel to the major drainage system and cut across different lithological boundaries of the study area. It is found from the map that major lineaments are trending NNW-SSE to NW-SE and some are NNE-SSW to NE-SW. Some small-scale lineaments are trending in the $\mathrm{N}-\mathrm{S}$ to the $\mathrm{E}-\mathrm{W}$ direction also.

Further analysis shows that the smaller lineaments form two different couple sets, set 1 which represents the majority trending $\mathrm{E}-\mathrm{W}$ and NW-SE, the second set trends N-S and NE-SW as indicated in the rose diagram (inset of figure 13). These two sets are actually represented as shear fractures (making a characteristic angle of $45^{\circ}$ between them) which might have occurred due to the vertical pushing by advancing magma dykes along the major lineaments/faults in the crustal level to form cinder cones.

The geometric control of cinder cones with the lineaments are further mapped by near analysis in the GIS platform. This analysis calculates a perpendicular distance between the cinder cones and the nearest lineament, where almost $80 \%$ cinder cones are situated within $2000 \mathrm{~m}$ from the lineaments (results in table 2). We thus consider such lineaments with their distances from cinder cones as characteristic features in HAB.

\section{Probable root plumbing system in the $\mathrm{HAB}$ and Asir foreland induced by volcano-structural analysis}

The structural setting of an active basaltic volcanic field along with the magnitude of the stress field are perhaps the most important criterion to be deciphered along the pathway through which the basaltic magma ascends from source to the earth surface. Plenty of case studies are known worldwide that help understanding the characteristics of the root plumbing system below the active volcanic provinces (Portal et al. 2013; Blaikie et al. 2014; Bolos et al. 2015). The final configuration of the pathway also depends on the nature of gravitational and tectonic forces operating there including the basal geometry, surface topography, stress changes that occurred during dyke propagation and finally to the stress barriers related to structural discontinuities (also refer Tibaldi et al. 2014; Bolos et al. 2015). Any inference on the root-plumbing system usually relies on: (i) a fair idea of the source of volcanic material and its differentiation in the crust-mantle zone and (ii) the knowledge regarding faults or the conduits for channelling the volcanic masses from the deeper crust to the surface, and the behaviour of masses of volcanic origin under the identified CGH, AGL and JSGH gravity anomaly zones.

In the Al Birk Basaltic field, specifically north of the Ad Darb transform fault (figure 3) contains alkali basaltic scoria and cinder cones that form extensive lava fields, lava domes and lava coulees. This field is developed from Pleistocene 


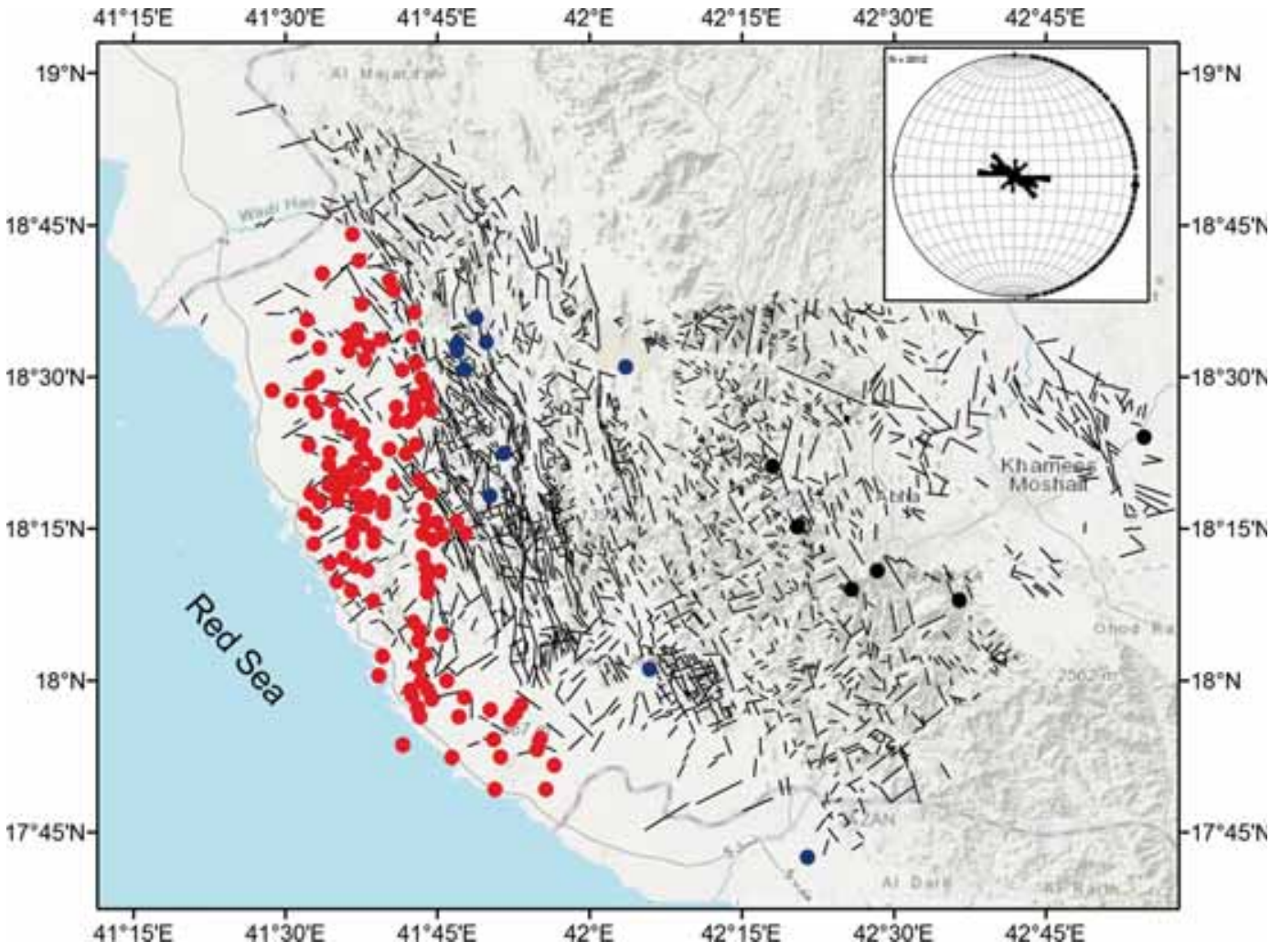

Figure 13. Lineaments/faults and fractures in the HAB and Asir foreland. Major feeder lineaments/faults are oriented, NNW-SSE to NW-SE, to form juvenile cinder cones. See also two conjugate shear couple sets, E-W and NW-SE, and N-S and NE-SW (in the inset rose diagram) generated due to vertical pushing produced by the magma movement. Red circles are cinder cones of CGH, blue circles for AGL and black circles for JSGH.

Table 2. Results of near analysis conducted on lineaments and cinder cones to understand the bearing between the two.

\begin{tabular}{lcc}
\hline $\begin{array}{l}\text { Distance range in } \\
\text { metres from lineaments } /\end{array}$ & $\begin{array}{c}\text { Number of cinder } \\
\text { cones within the } \\
\text { faults/fractures }\end{array}$ & $\begin{array}{c}\text { Cumulative \% of } \\
\text { cinder cones within } \\
\text { the distance range }\end{array}$ \\
\hline $0-250$ & 15 & 10.00 \\
$250-500$ & 15 & 20.00 \\
$500-750$ & 15 & 30.00 \\
$750-1000$ & 23 & 45.33 \\
$1000-1250$ & 16 & 56.00 \\
$1250-1500$ & 15 & 66.00 \\
$1500-1750$ & 8 & 71.33 \\
$1750-2000$ & 12 & 79.33 \\
$2000-2250$ & 10 & 86.00 \\
$2250-2500$ & 8 & 91.33 \\
$2500-2750$ & 3 & 93.33 \\
$2750-3000$ & 3 & 95.33 \\
$3000-3250$ & 1 & 96.00 \\
$3250-3500$ & 1 & 96.67 \\
$3500-3750$ & 0 & 96.67 \\
$3750-4000$ & 1 & 97.33 \\
$>4000$ & 4 & 100.00 \\
\hline
\end{tabular}

The first column represents the perpendicular distance range between the cinder cones with the edge of the lineaments, the second and third columns represent the frequency and cumulative frequency percentage, respectively. 
to the present (Hadley 1982; Brown et al. 1989; Németh et al. 2014). In fact, they are equivalent of the sub-alkaline tholeiitic basalt of the present day axial rift of the Red Sea but represent small restricted flows. The alkali basalts were erupted through a thicker Precambrian crust, approximately $47-\mathrm{km}$ thick, and represented as mixtures of harzburgites, cumulate gabbro and websterite inclusions of $4-20 \mathrm{~cm}$ in size from the mantle source and also accompanied by large $(>2 \mathrm{~cm})$ megacrysts of glassy alumina-rich clinopyroxene, plagioclase and spinel of the probable crustal origin (Hadley 1982). The harzburgites, websterites and cumulate gabbros as inclusion in alkali basalt reveal composition variations and are related to a complex mantle history, whereas clinopyroxene and plagioclase megacrysts may represent the early phases that crystallised from the alkali olivine basalt magma at depths less than $35 \mathrm{~km}$ (Hadley 1982). The abundant crustal lithic- and mantlederived nodules suggest significant excavation and cratering (Németh et al. 2014). Based upon the available data, the plagioclase megacrysts formed at depths not greater than that of the lower crust, but the clinopyroxene megacrysts could have crystallised under upper mantle pressure and temperature condition. The occurrence of cumulus textures and layering within these mantle-derived inclusions suggest that they are crystallised during the period when the alkaline magma was not rapidly ascending. The harzburgites are of refractory category and extracted from depleted mantle material. Hence, the host basalt magmas of HAB must have been derived from un-depleted mantle at a greater depth. Calculations of inferred pressures of equilibration of basaltic magma with mantle mineral assemblages suggest that the depleted harzburgite inclusions came from depths less than about $70 \mathrm{~km}$, pressures less than 20-24 kb (Hadley 1982). TGDZ (figure 5; also referred to as tcd in figure 3) is represented as a dyke swarm with an average width of $\sim 20 \mathrm{~km}$. These sheeted dykes are composed of gabbro (with clinopyroxene, plagioclase with accessory magnetite, chlorite and apatite with \pm hornblende), yielded an age of 19-27 Ma (K/Ar whole rock age), and are emplaced close to the initial opening of the Red Sea rift (Hadley 1982). These parallel normal faults along the sheeted dyke zones are generally north to the north-west trend, and in our analysis, these normal faults and subsidiary fault zones within the crustal level act as major feeder channels (as stated in section 5) for the volcanoes of the CGH, AGL and JSGH zones.
In gravity modelling, the total isostatic gravity correction is made using the plate-tectonic system out-to-a-radius of $166.7 \mathrm{~km}$ centring the gravity station, and the combined topographic-isostatic correction beyond that distance, as discussed by Karki et al. (1961). Furthermore, the calculation of the residual isostatic gravity anomaly (IGA) is accomplished in three stages. IGA1 is calculated based on the plate-tectonic system. IGA2 is derived after the removal of the regional gravity gradient. Finally, IGA3 (residual IGA) is calculated after the removal of the regional gravity gradient and correction for the model geologic provinces (see Gettings 1984 for further details).

To understand the masses of volcanic origin under the respective gravity anomaly zones (CGH, AGL and JSGH), we have generated a gravity profile (figure 14a) along the traverse $\mathrm{A}^{\prime}$ and $\mathrm{B}^{\prime}$ of figure 5 . The BA, along with $\mathrm{CGH}$, shows an anomaly amplitude from +20 mgal, and exhibiting steep gravity gradient towards AGL with a fall to $-100 \mathrm{mgal}$, followed by a general upward highamplitude flexure up to $-60 \mathrm{mgal}$ in the JSGH zone. The free-air (FA) and IAG3 show moderately low values in $\mathrm{CGH}$, exhibiting a moderate gravity gradient towards AGL and a very steep gravity gradient and very high-amplitude peak along JSGH. The overall trend in gravity data indicates that in $\mathrm{CGH}$, the volcanic mass load is moderate (due to the material load of $\sim 200$ small but prominent volcanic cones) with a general reduction of mass towards AGL (with possible fewer volcanic material), and an extraordinary increase in mass towards JSGH due to a contrasting change in elevation, underplating and accumulation of more crustal/volcanic material. We infer that the major source area of volcanic material is in JSGH that hosts the main large cinder cones. In JSGH, IAG3 gravity highs may be correlated with intruded and accreted volcanic material, whereas intermediate to low gravity anomalies of IAG3 occurring in CGH and AGL indicate an overall volcanic section. Moreover, the high wavelength anomalies in JSGH with a broad central gravity high illustrated in BA, FA and IAG3 data may indicate density contrasts whose sources lie entirely within the crust having anomalous geochemical characteristics and thermal state.

In order to gain an understanding of the stress field operating across the region, we study the structural pattern and orientation of the major faults. Here, the zone of normal faulting is clearly represented as a sheeted dyke system that indicates 
(a)

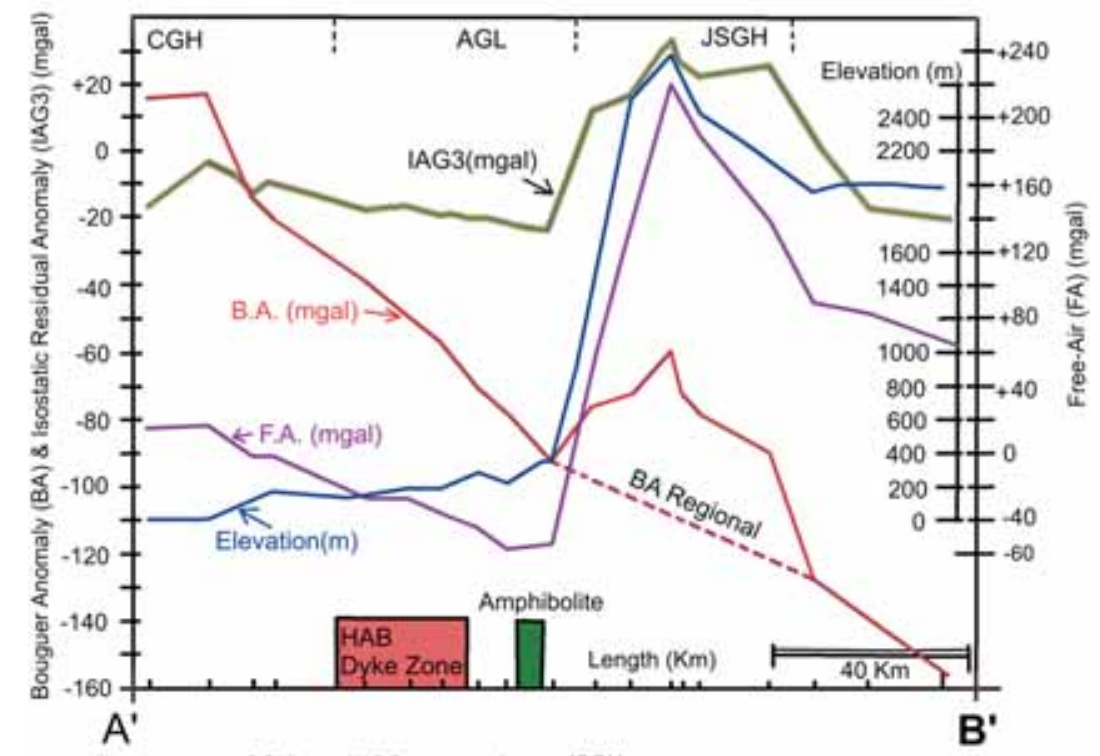

(b)

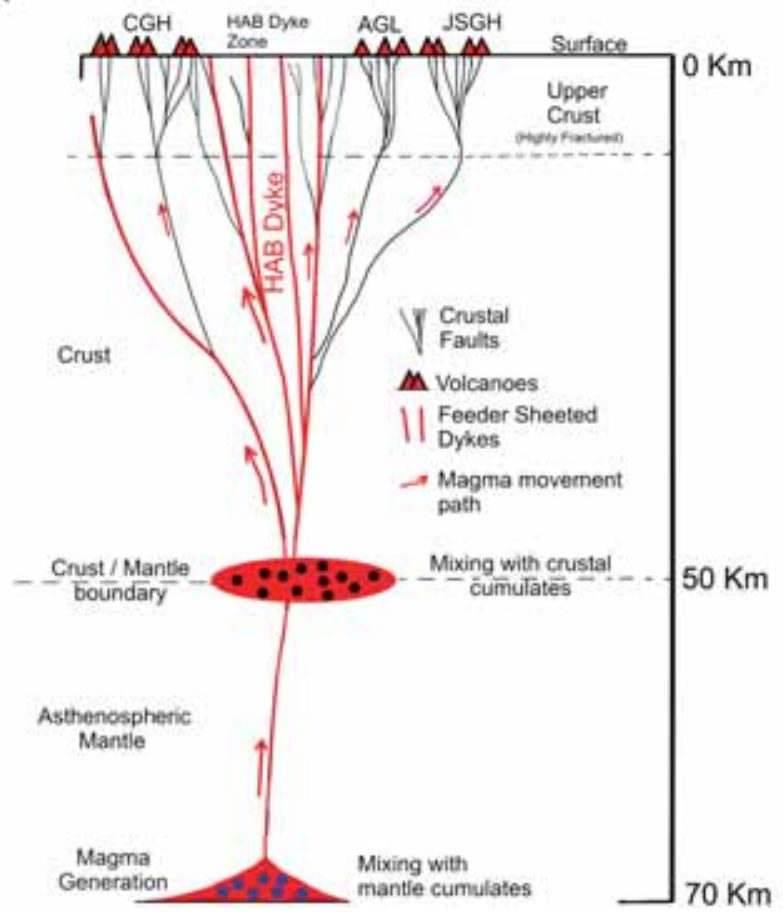

Figure 14. (a) 2D-gravity profile for the traverse $\mathrm{A}^{\prime}-\mathrm{B}^{\prime}$ (of figure 5) across the zones of CGH, AGL and JSGH. (b) Illustration of the source model for volcanic masses and the probable root-plumbing system under the harrat.

a dominant extension $\left(\sigma_{3}\right)$ along the NE-SW direction, intermediate axis $\left(\sigma_{2}\right)$ along the NW$\mathrm{SE}$ direction and a vertical compression axis $\left(\sigma_{1}\right)$. The major extension has resulted in the generation of space in the upper crust for the magma movement and as an aid to the formation of a new fault and conjugate shear fracture systems in the rifted crust.

This allows us to introduce a conceptual mechanism on probable volcano root-plumbing system of the area (figure 14b). The proposed model invokes that the alkali basaltic magma in HAB is generated at the lithospheric depth $(\sim 70 \mathrm{~km})$ at a pressure less than 20-24 kb pressure where it collects the mantle cumulates in the form of harzburgites, websterites and cumulate gabbros. On its upward journey, the magma accumulates the plagioclase and clinopyroxene megacrysts that have crystallised under the upper mantle/crust-mantle boundary pressure and temperature condition. Within the crust, the magma follows natural conduits that existed as zone of parallel normal fault 
systems within the HAB dyke zone, and subsequently created several crustal faults and shear fractures by hydro-fracturing. These newly created faults/fractures had eventually generated a new pathway for magma emplacement and produced prominent volcanoes in the JSGH zones with juvenile craters mainly along CGH. The presentday volcanic material supply is more towards CGH which gives the juvenile cinder cones. The gravity anomalies in FA and IAG3 along the JSGH zone (figure 14a) indicate that this zone acts as a probable source of volcanic material, and also yielded prominent and large cinders in the Al-Birk and Asir mountains foreland. In this model, the TGD zone acts as a vertical magma sheet which offers the main volcanic supply channel.

\section{Summary and discussion}

The HAB contains alkali basaltic volcanoes (scoria and spatter cones) from the extensive lava fields developed from Pleistocene to the present (Hadley 1982; Brown et al. 1989; Németh et al. 2014). We have identified for the first time that the cinder cones occur in three distinct BA gravity zones as $\mathrm{CGH}$, an AGL and a sinuous gravity high further east named as JSGH. In my opinion, these gravity anomaly zones indicate crustal heterogeneity in a high-density transitional crust.

Interpreted satellite imagery for 150 sizeable prominent cinder cones situated within CGH, AGL and JSGH are subdivided according to gravity anomaly zones are 134 (90\%) in CGH, 10 (6\%) in AGL and $6(4 \%)$ in JSGH. We identify for the first time that the juvenile cinder cones (age $<1 \mathrm{Ma}$ ) are characterised by sub-vertical slope angles $\left(<40^{\circ}\right)$. The morphometric measurements of 150 cinder cones belonging to CGH, AGL and JSGH show that cone height $\left(H_{\mathrm{co}}\right)$ is 0.07 times the cone basal diameter $\left(W_{\mathrm{co}}\right)$ for $\mathrm{CGH}$, and 0.08 for AGL and that for JSGH is 0.12 . This result is near the regression obtained globally as $H_{\text {co }}=0.18 W_{\text {co }}$ (see Wood 1980a). The median basal diameter $\left(W_{\text {co }}\right)$ for 150 measured cinder cones is $0.918 \mathrm{~km}$ and varies between 0.41 and $9.6 \mathrm{~km}$. This measurement is tallying well with the measured global median of $W_{\text {co }}$ for the cinder cones of $0.8 \mathrm{~km}$ (Wood 1980a), which also demonstrate the accuracy of the measurements in the present study. The basal diameter of the cinder cone ranges: $1.19-9.60 \mathrm{~km}$ with an average of $3.68 \mathrm{~km}$ in JSGH, $0.45-1.97 \mathrm{~km}$ with an average of $0.84 \mathrm{~km}$ in AGL and $0.4-2.27 \mathrm{~km}$ with an average of $0.96 \mathrm{~km}$ for the $\mathrm{CGH}$ zone. Considering these calculated values, the depth of the magma chamber of AGL and CGH varies between 3 and $35 \mathrm{~km}$ but mostly placed less than $20 \mathrm{~km}$ depth. In contrast, the chamber of the older cinder cones of JSGH was deep-rooted with an average depth of 40 $50 \mathrm{~km}$ (see Fedotov et al. (1976a, b) for more insight into the calculation of the depth of the magma chamber).

The crater diameter $\left(W_{\mathrm{cr}}\right)$ is 0.19 times the cone basal diameter $\left(W_{\text {co }}\right)$ for CGH, 0.15 times for AGL and 0.2 times for JSGH. The degradation of cinder cones with age, its response to the rate of erosion is mapped. JSGH is the oldest among the three volcanic zones with a high $H_{\mathrm{co}} / W_{\text {co }}$ ratio and the cones of JSGH are characterised by angular asymmetry at their base angles. Furthermore, the slope angle provides information about the age of scoria edifices or cones, the greater the slope angle higher the age. In the JSGH area, the average slope is the highest and varies from $27^{\circ}$ to $40^{\circ}$, whereas for $\mathrm{AGL}$, it is $25^{\circ}$ and for the CGH area, it is $2^{\circ}-15^{\circ}$. Moreover, the $H_{\mathrm{co}} / W_{\text {co }}$ ratio is 0.02 times the volume for JSGH, whereas this is comparatively very low (0.0008) for CGH and 0.01 times for AGL, indicating the morphological slope for the oldest JSGH is steeper compared to the slope along AGL and CGH.

It is found that the major lineaments are oriented NNW-SSE to NW-SE and the minor lineaments form two different shear couple sets, majority trending $\mathrm{E}-\mathrm{W}$ and $\mathrm{NW}-\mathrm{SE}$, and secondary trends N-S and NE-SW. As per our structural analysis, these two sets of minor lineaments are represented as conjugate shear fractures generated due to vertical pushing (along the $\sigma_{1}$ axis) produced by the advanced magma movement in the lower crust from the upward-arching mantle along the major feeder lineaments/faults oriented NNWSSE to the NW-SE, to form juvenile cinder cones. It is also interesting to summarise that within a distance of $2000 \mathrm{~m}$ from the lineaments, $79.33 \%$ of the cinder cones lie, which indicates the average distance under which the feeder lineaments supply volcanic material to the cinder cones.

The structural patterns also shown by the eruptive centres and the orientation of major lineaments suggests an overall extension $\left(\sigma_{3}\right)$ along the NE-SW direction. This extension has resulted in the generation of parallel gravity faults of long dimensions along the NW-SE direction in tertiary times, and they are represented, in the present 
day, on the map as the sheeted dyke system. This zone represents the major rifting axis operating since the tertiary period parallel to the presentday extensional axis along the Red Sea. The cones and craters are also disposed along the NW-SE trend parallel to the feeding system, a similar result has also been observed in the La Garrotxa Volcanic Field in NE Iberia by Bolos et al. (2015).

The volcano-structural analysis also indicates that the alkali basaltic magma of the harrat is generated at about $70-\mathrm{km}$ depth at a pressure of less than 20-24 kb in the asthenospheric mantle where the mantle cumulates, and later the plagioclase and clinopyroxene megacrysts from the crust-mantle boundary nearly at $40-50 \mathrm{~km}$ depth. Upon reaching the crust, it takes the natural conduits like the normal faults existing in the HAB dyke zone (TDG zone), and created several crustal lineaments/faults/fracture as the conjugate shear systems by hydro-fracturing to form volcanoes in JSGH with juvenile craters mainly along CGH. In this model, the TGD zone acts as vertical magma sheets which offer the main volcanic supply channel. These results, when integrated to surface geological maps and gravity profiles, provide a probable indication on the root-plumbing system of the volcano edifice and are presented in figure 14 . We conjecture that the basalts in $\mathrm{HAB}$ are the produce of the volcanic process from the source area in JSGH that hosts the major cinders. In this model, TGDZ acting as vertical magma sheets largely contributed to the development of the cinder cones. The earthquakes generated in this volcanic field yield a low $b$ value which connotes mainly for their extensional tectonic origin rather than of volcanic origin.

\section{Conclusions}

The following conclusions can be drawn from the present study:

(i) The cinder cones of $\mathrm{HAB}$ occur in three distinct BS zones CGH, AGL and JSGH. These three gravity anomaly zones indicate zones of distinct crustal heterogeneity within a wide transitional crust of higher density in the study area.

(ii) Morphometric measurements indicate that the juvenile cinder cones (age $<1 \mathrm{Ma}$ ) are characterised by sub-vertical slope angles $\left(<40^{\circ}\right)$. The morphological slope for the oldest JSGH is steeper compared to the slope along AGL and CGH. The depth of the magma chamber of AGL and $\mathrm{CGH}$ is less than $20 \mathrm{~km}$, whereas, the chamber of the older cinder cones of JSGH is deep-rooted with an average depth of $40-50 \mathrm{~km}$. About $79.33 \%$ of the cinder cones lie within a distance of $2 \mathrm{~km}$ from the feeder lineaments that supply volcanic material to the volcanoes. Our measurement (table 1) also illustrates that a minimum of $41376.64 \times 10^{6} \mathrm{~m}^{3}$ of erupted basaltic lava presently forms the cinder cones in HAB.

(iii) The volcano-structural analysis and root plumbing model indicates that the alkali basaltic magma is generated at about $70 \mathrm{~km}$ depth at a less than 20-24 kb pressure in the asthenospheric mantle. On its upward journey up through crust, the magma has created several feeding channels (new crustal lineaments/faults/conjugate fractures) to form volcanoes in JSGH zones with juvenile craters mainly along $\mathrm{CGH}$ in recent times. TGDZ acts as vertical magma sheets which offer the main volcanic supply channel. The earthquakes spawned in HAB are of extensional tectonic origin rather than of volcanic origin.

\section{Acknowledgements}

The Editor-in-Chief, Prof N V Chalapathi Rao and anonymous reviewers are warmly acknowledged for their useful comments. Manoj Mukhopadhyay acknowledges the support from the ISPP deanship of the visiting professor, King Saud University, Riyadh, while doing this work.

\section{References}

Abdelfattah A K, Al-Amri A, Abd el-aal A K, Zaidi F K, Almadani S and Al-Arifi N 2017 The 23 January 2014 Jizan earthquake and its tectonic implications in southwestern Saudi Arabia; Tectonophys. 712-713 494-502.

Aki K 1965 Maximum likelihood estimate of $b$ in the formula $\log N=a-b M$ and its confidence limits; Bull. Earthq. Res. Inst., Tokyo Univ. 43 237-239.

Blaikie T N, Ailleres L, Betts P G and Cas R A F 2014 A geophysical comparison of the diameters of simple and complex maar volcanoes, Newer Volcanics Province, south-eastern Australia; J. Volcanol. Geotherm. Res. 276 64-81.

Bohannon R G, Naeser C W, Schmidt D G and Zimmwermann R G 1989 The timing of uplift, volcanism and 
rifting peripheral to the Red Sea, a case for passive rifting; J. Geophys. Res. 94 1683-1701.

Bolos X, Marti J, Becerril L, Planaguma' L, Grosse P and Barde-Cabusson S 2015 Volcano-structural analysis of La Garrotxa Volcanic Field (NE Iberia): Implications for the plumbing system; Tectonophys. 642 58-70.

Bosworth W, Huchon P and McClay K 2005 The Red Sea and Gulf of Aden Basins; J. Afr. Earth Sci. 43 334-378.

Brown G F, Schmidt D L and Huffan Jr A C 1989 Geology of the Arabian Peninsula, shield area of western Saudi Arabia; US Geological Survey Professional Paper 560-A, $188 \mathrm{p}$.

Coleman R G, Gregory R T and Brown G F 1983 Cenozoic volcanic rocks of Saudi Arabia; US Geological Survey Open-File Report OF-03-83, pp. 1-82.

Devès M, Inglis R, Meredith-Williams M, Al Ghamdi S, Alsharekh A M and Bailey G N 2013 Paleolithic survey in Southwest Saudi Arabia: Methodology and preliminary survey; Adumatu 27 7-30.

Fedotov S A, Kherenov A P and Chirkov A M 1976a Seismologic data on magma chambers, mechanism and evolution of 1975 Tolbachik basalt fissures erupted on Kamchatka; Dokl. Akad. Nauk SSSR 228 93-96.

Fedotov S A, Kherenov A P and Chirkov A M 1976b The great 1975 fissure eruption of Tolbachik, Kamchatka; Dokl. Akad. Nauk SSSR 228 87-89.

Gettings M E 1984 The isostatic gravity anomaly field of southwestern Saudi Arabia and its interpretation; Technical Record USGS-TR-04-22, Ministry Petrol. Mineral Resources, Jiddah, Kingdom of Saudi Arabia, 107p.

Gettings M E, Blank Jr H R, Mooney W D and Healey J H 1986 Crustal structure of southwestern Saudi Arabia; J. Geophys. Res. 91(B6) 6491-6512.

Ghent E D, Coleman R G and Hadley D G 1980 Ultramafic inclusions and host alkali basalts of the southern coastal plain of the Red Sea, Saudi Arabia; Am. J. Sci. 280-A 499-527.

Hadley D G 1982 Reconnaissance geology of the Jabal Hashahish Quadrangle 17/1418, kingdom of Saudi Arabia; US Geological Survey Open-File Report 82-286, pp. $1-14$.

Healy J H, Mooney W D, Blank H R, Gettings M E, Kohler W M, Lamson R J and Leone L E 1982 Saudi Arabian seismic deep-refraction profile; Final Project Report, Open-File Report USGS-OF-02-37, pp. 1-429 (including appendices, Saudi Arabia Deputy Minist. Miner. Resour., Jiddah).

Hooper D M and Sheridan M F 1998 Computer-simulation models of scoria cone degradation; J. Volcanol. Geoth. Res. 83 241-267.

Inglis R H, Sinclair A G M, Shuttleworth A, Alsharekh A M and Al Ghamdi S 2013 Preliminary report on 2013 fieldwork in Southwest Saudi Arabia by the DISPERSE project: Jizan and Asir Provinces, February-March 2013; Unpublished Report, Saudi Commission for Tourism and Antiquities.

Inglis R H, Sinclair A, Shuttleworth A, Alsharekh A, Al Ghamdi S, Devès M, Meredith-Williams M G and Bailey G N 2014a Investigating the palaeolithic landscapes and archaeology of the Jizan and Asir regions, Southwest Saudi Arabia; Proc. Semin. Arab. Stud. 44 193-212.
Inglis R H, Sinclair A G M, Shuttleworth A, Al Maamary A, Budd W, Hausmann N, Meredith-Williams M G, Alsharekh A M, Al Ghamdi S and Bailey G N 2014b Preliminary report on 2014 fieldwork in SW Saudi Arabia by the DISPERSE project: Jizan and Asir provinces. DISPERSE 2014 Report, University of York, UK, 31p, http://eprints.whiterose.ac.uk/80965/.

Karki P, Kivioja L and Heiskanen W A 1961 Topographicisostatic maps for the world for the Hayford zones 18-1, Airy-Heiskanen system, $T=30 \mathrm{~km}$, Vol. 35; International Association of Geodesy, Isostatic Institute Publication, 5 p.

Kereszturi G and Németh K 2012a Monogenetic basaltic volcanoes: Genetic classification growth geomorphology and degradation; In: Updates in volcanology - New advances in understanding volcanic systems (ed.) Németh K, IntertechOpen, 89p, https://doi.org/10.5772/51387, ISBN: 978-953-51-0915-0.

Kereszturi G and Németh K 2012b Structural and morphometric irregularities of eroded Pliocene scoria cones at the Bakony-Balaton Highland volcanic field, Hungary; Geomorphology 136(1) 45-58.

Kereszturi G, Jordan G, Németh $\mathrm{K}$ and Dóniz-Páez J F 2012 Syn-eruptive morphometric variability of monogenetic scoria cones; Bull. Volcanol. 74(9) 21712185 .

Merghelani H M and Gallanthine S K 1980 Microearthquakes in the Tihamat Asir region of Saudi Arabia; Bull. Seism. Soc. Am. 70(6) 2291-2293.

Mogren S and Mukhopadhyay M 2013 Gravity modeling for the rifted crust at the Arabian shield margin - Further insight into Red Sea spreading; Open J. Geol. 3 $28-33$.

Mooney W D, Gettings M, Blank H R and Healy J H 1985 Saudi Arabian seismic deep-refraction profileA travel time interpretation of deep crustal structure; Tectonophys. 111(3-4) 173-246, https://doi.org/10. 1016/0040-1951(85)90287-2.

Németh K, Moufti M R, El-Masry N, Qaddah A and Pécskay Z 2014 Maars over cones: Repeated volcanism in the same location along fissures in western Saudi Arabian volcanic fields; In: IAVCEI - 5IMC, Querétaro, México, November 2014, pp. 2-3.

Portal A, Labazuy P, Lénat J F, Béné S, Boivin P, Busato E, Cârloganu C, Combaret C, Dupieux P, Fehr F, Gay P, Laktineh I, Miallier D, Mirabito L, Niess V and Vulpescu B 2013 Inner structure of the Puy de Dôme volcano: Cross-comparison of geophysical models (ERT, gravimetry, muon imaging); Geosci. Instrum. Methods Data Syst. $247-54$.

Schimdt D L, Hadley D G and Brown G F 1983 Middle tertiary continental rift and evolution of the Red Sea in southwestern Saudi Arabia; USGS Open File Report 83641, pp. 1-60.

Taachikawa T, Kaku M and Iwasaki A 2011 ASTER GDEM, version 2; Validation report, https://ssl.jspacesystems. or.jp/library/archives/ersdac/GDEM/ver2Validation/ Appendix_A_ERSDAC_GDEM2_validation_report.pdf.

Tibaldi A, Bonali F L and Corazzato C 2014 The diverging volcanic rift system; Tectonophys. $61194-$ 113. 
Wood C A 1980a Morphometric evolution of cinder cones; J. Volcanol. Geoth. Res. 7 387-413.

Wood C A 1980b Morphometric analysis of cinder cone degradation; J. Volcanol. Geoth. Res. 8 137160 .

Corresponding editor: N V ChALAPATHI RAO
Zaidi F K and Mukhopadhyay M 2015 Morphometric analysis of the scoria cones and drainage pattern for the Quaternary and older volcanic fields in parts of the Large Igneous Province, Saudi Arabia; J. African. Earth Sci. $1101-13$. 